\title{
To snake or not to snake in the planar Swift-Hohenberg equation
}

\author{
Daniele Avitabile \\ Department of Engineering Mathematics \\ University of Bristol \\ Bristol, BS8 1TR, UK \\ John Burke \\ Department of Mathematics and Statistics \\ Boston University \\ Boston, MA 02215, USA
}

\author{
David J.B. Lloyd \\ Department of Mathematics \\ University of Surrey \\ Guildford, GU2 7XH, UK \\ Edgar Knobloch \\ Department of Physics \\ University of California at Berkeley \\ Berkeley, CA 94720, USA
}

\author{
Björn Sandstede \\ Division of Applied Mathematics \\ Brown University \\ Providence, RI 029012, USA
}

April 9, 2010

\begin{abstract}
We investigate the bifurcation structure of stationary localised patterns of the two-dimensional SwiftHohenberg equation on an infinitely long cylinder and on the plane. On cylinders, we find localised roll, square and stripe patches that exhibit snaking and non-snaking behaviour on the same bifurcation branch. Some of these patterns snake between four saddle-node limits: recent analytical results predict then the existence of a rich bifurcation structure to asymmetric solutions, and we trace out these branches and the PDE spectra along these branches. On the plane, we study the bifurcation structure of fully localised roll structures, which are often referred to as worms. In all the above cases, we use geometric ideas and spatial-dynamics techniques to explain the phenomena we encounter.
\end{abstract}

\section{Introduction}

Stationary spatially localised patterns are of great interest to both experimentalists and theorists [15, 33]. Such structures are observed in reaction-diffusion systems [28], in optical systems [7,39], electrical discharges [2], liquid crystals [24, 32] and even in ferrofluids [35]. Related but generally time-dependent structures have been observed in several different fluid flows, including binary fluid convection [26] and plane Couette flow $[16,42]$. Time-periodic localised states called oscillons were first observed in parametrically driven granular media [44] and subsequently in parametrically driven liquids [1, 29]. In many of these systems, the structures form bound states due to mutual attraction, often with an underlying hexagonal structure $[1,2,7]$; when parameters are varied, these structure may dissociate or melt [7].

All these systems are nominally homogeneous, and the localised structures form in regimes in which a spatially homogeneous trivial state coexists with a spatially structured state. Indeed, existing theory suggests that many if not all such states are associated with the presence of a subcritical Turing bifurcation, i.e., a pattern-forming instability of the trivial state. In $\mathbb{R}^{2}$ such a bifurcation creates localised states, in addition to regular patterns such as stripes, squares and hexagons. Numerical continuation indicates that near this bifurcation the localised structures resemble a regular pattern with a superimposed broad, slowly decaying 


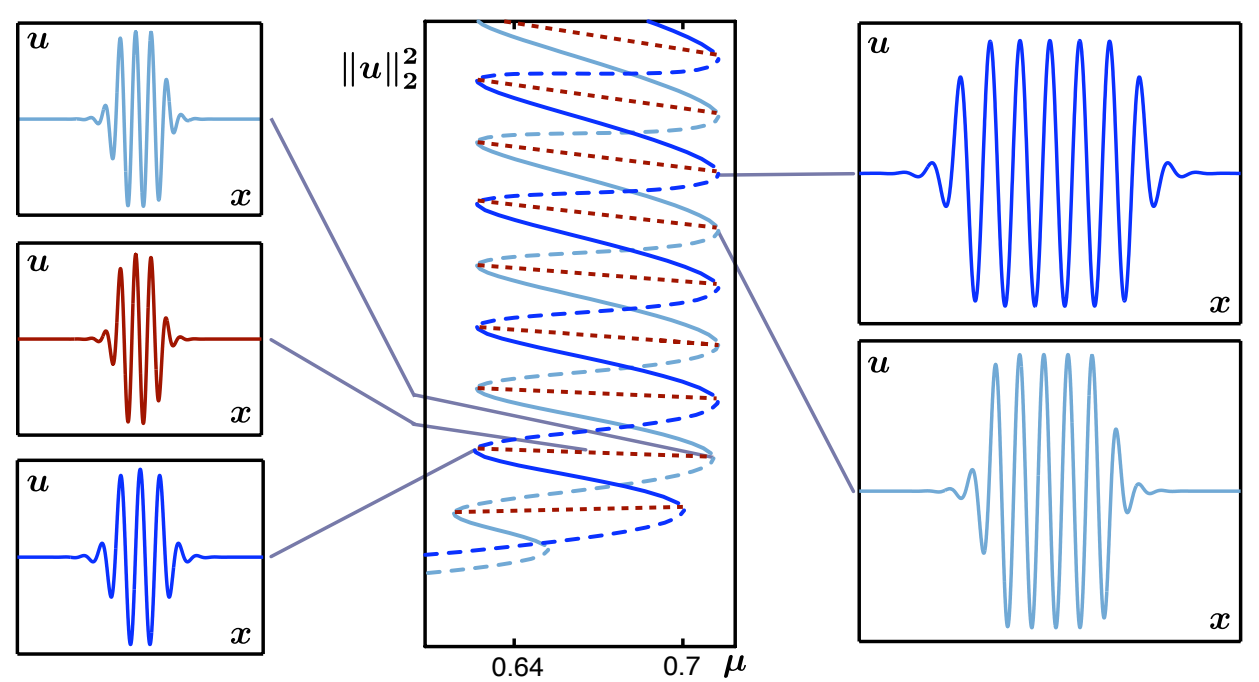

Figure 1: Snakes-and-ladders bifurcation diagram of localised patterns of (1.1) for $\nu=2$. The localised structures are stable along solid branches and unstable along dashed branches.

envelope: in particular, there is no well-defined interface between the regular pattern in the interior of the localised structure and the surrounding homogeneous background state. However, in many cases this interface sharpens significantly as an appropriate parameter is varied, so that the localised structure acquires a well-defined width. With further variation of the parameter, the localised structures begin to widen in space, and it is this phenomenon, and the underlying mechanisms which cause it, that we investigate in this paper. We employ for this purpose a model equation, the Swift-Hohenberg equation

$$
u_{t}=-(1+\Delta)^{2} u-\mu u+\nu u^{3}-u^{5}, \quad \mathbf{x} \in \mathbb{R}^{d}, \quad d=1,2 .
$$

This equation describes pattern-forming systems near instabilities with nontrivial finite spatial wavelength $[15,21]$ but has variational dynamics with energy

$$
\mathcal{E}(u)=\int_{\mathbb{R}^{d}}\left[\frac{[(1+\Delta) u]^{2}}{2}+\frac{\mu u^{2}}{2}-\frac{\nu u^{4}}{4}+\frac{u^{6}}{6}\right] \mathrm{d} \mathbf{x} .
$$

Local minima of $\mathcal{E}$ correspond to stable stationary solutions of (1.1). While the physical examples motivating our work are in general non-variational, it turns out that the Swift-Hohenberg equation sheds a great deal of light on the growth of stationary spatially localised structures in general.

We begin by briefly summarizing the situation in one space dimension before outlining the new phenomena that we expect to encounter in two dimensions. Figure 1 shows the bifurcation diagram of stationary solutions of (1.1) on $\mathbb{R}$ computed in [8-10]. There are two intertwined snaking branches corresponding to even and odd localised structures. As we move upwards on these branches, the localised structures widen by acquiring additional periodic rolls at an infinite number of successive fold bifurcations [13, 45]. Near each fold, the localised patterns undergo a pitchfork bifurcation at which a horizontal ladder branch of asymmetric stationary structures emerges that connects the two intertwined snaking branches. As the localised roll patterns become wider, they resemble stationary bound states of fronts that connect the homogeneous background state to a regular periodic pattern. Physically, the origin of the resulting snaking behaviour can be traced to the presence of a "pinning potential" between the fronts and the periodic state between them [34]. The presence of this potential implies that the fronts will remain stationary as a parameter is varied until the energy difference between the roll state and the homogeneous background overcomes the pinning potential. In contrast, stationary fronts connecting the background to another homogeneous steady state are expected to exist at isolated parameter values only: in this case there is no pinning potential and an arbitrarily small change in the parameter will cause each front to move. A "bound" state of two such fronts will therefore either expand or shrink, except at isolated parameter values. 


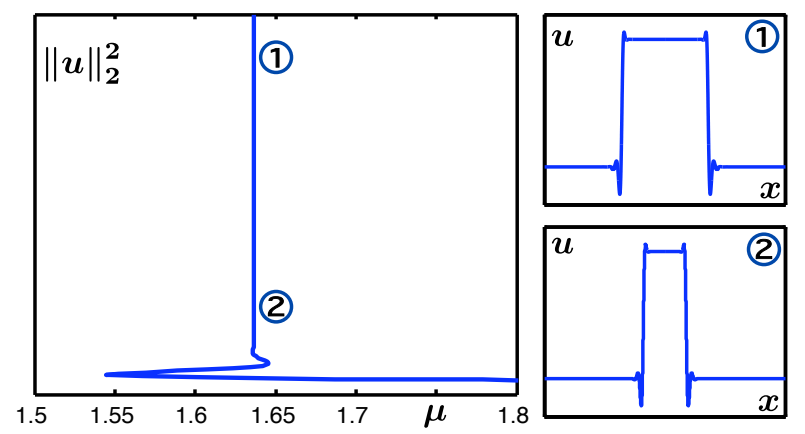

Figure 2: Non-snaking of localised pulses of (1.1) for $\nu=3.75$. The broadening of the pulse is reflected in an increase of its $L^{2}$-norm.

Rephrased in dynamical systems language for the steady-state equation

$$
\left(1+\partial_{x}^{2}\right)^{2} u+\mu u-\nu u^{3}+u^{5}=0, \quad x \in \mathbb{R}
$$

associated with (1.1), we can identify the localised roll patterns with homoclinic orbits that bifurcate from a heteroclinic cycle which corresponds to the aforementioned pair of fronts. The heteroclinic orbits in the heteroclinic cycle connect an equilibrium, corresponding to the homogeneous rest state, to a periodic orbit which corresponds to the underlying periodic roll pattern. Thus, if we assume the existence of these fronts, we should be able to predict the snaking and ladder branches using homoclinic bifurcation theory, and such an analysis has indeed been carried out recently in [5]. For the quadratic-cubic Swift-Hohenberg equation the existence of both fronts and localised roll structures, together with their snaking scenario, has been established using a formal beyond-all-orders asymptotic analysis for $(\mu, \nu)$ close to zero [12, 27].

We may also consider localised structures that are bound states of fronts that connect the homogeneous background state to another homogeneous state, instead of to a periodic structure. The associated spatialdynamics scenario involves the bifurcation of homoclinic orbits from two heteroclinic orbits that connect two equilibria to each other. This scenario was investigated in [25], and the resulting bifurcation curve associated with localised structures collapses to a single asymptote in parameter space, rather than oscillating back and forth between two asymptotes. As already mentioned, this is a consequence of the absence of a pinning potential. We refer to these bifurcation diagrams as non-snaking scenarios; see Figure 2.

The goal of this paper is two-fold. First, the results in [5] suggest that snakes \& ladder bifurcation structures can potentially be far more complicated than those found in [9] for the one-dimensional Swift-Hohenberg equation. It turns out that these more complicated scenarios occur in the planar Swift-Hohenberg equation posed on a cylinder, and we investigate the resulting patterns numerically in this paper. One particular, and perhaps surprising, finding is that asymmetric structures can be stable. Our second goal is to further elucidate the dichotomy between snaking and non-snaking for planar structures. While the different behaviours can be predicted for 1D structures using only their profiles, the distinction is more subtle for planar patterns, and it is our goal to show that it is, in general, not possible to predict whether a given pattern snakes or not by inspecting its profile.

The patterns we study on cylinders were first found in [9]: they resemble stripe patterns that evolve spatially towards spots or squares before converging to the homogeneous rest state. We also investigate fully localised stripe patterns on the plane that are often referred to as worms and present a formal spatial dynamics arguments for their bifurcation behaviour. We mention that localised patterns on the plane and on cylinders were investigated in [30] by numerical continuation. In [30], it was also shown how localised patterns of the Swift-Hohenberg equation on cylinders can be captured as homoclinic orbits of an appropriate reversible Hamiltonian spatial-dynamical system.

The outline of the paper is as follows. In $\S 2$, we set up the spatial dynamics formulation of the SwiftHohenberg equation and review some of the results about localised structures. The numerical algorithms we 
use are discussed in $\S 3$, and we present our numerical results in $\S 4$ and $\S 5$. In $\S 6$, we discuss open problems and comment on potential applications.

\section{Spatial dynamics}

In this section, we consider the stationary planar Swift-Hohenberg equation

$$
\left(1+\partial_{x}^{2}+\partial_{y}^{2}\right)^{2} u+\mu u-\nu u^{3}+u^{5}=0, \quad(x, y) \in S^{1} \times \mathbb{R}
$$

on the cylinder $S^{1} \times \mathbb{R}$, where $S^{1}=\mathbb{R} / 2 L_{x} \mathbb{Z}$ for some number $L_{x}>0$. Equation (2.1) captures all stationary planar patterns of (1.1) that are either periodic with period $2 L_{x}$ in the $x$-variable or else localised in the $x$-direction upon choosing $L_{x} \gg 1$. To explain the bifurcation structure of such patterns, which will also be localised in the $y$-direction, from a spatial dynamical systems viewpoint, we write (2.1) as a first-order system in $y$ and obtain

$$
U_{y}=\mathcal{A}(\mu) U+\mathcal{N}(U ; \nu), \quad U=\left(u, u_{y}, u_{y y}, u_{y y y}\right)^{t} \in \mathcal{X}
$$

where

$$
\mathcal{A}(\mu)=\left(\begin{array}{cccc}
0 & 1 & 0 & 0 \\
0 & 0 & 1 & 0 \\
0 & 0 & 0 & 1 \\
-\left(1+\partial_{x}^{2}\right)^{2}-\mu & 0 & -2\left(1+\partial_{x}^{2}\right) & 0
\end{array}\right), \quad \mathcal{N}(U ; \nu)=\left(\begin{array}{c}
0 \\
0 \\
0 \\
\nu U_{1}^{3}-U_{1}^{5}
\end{array}\right)
$$

and $U(y)$ is, for each fixed $y$, a function of $x$ that $\operatorname{lies}^{1}$ in $H^{3}\left(S^{1}\right) \times H^{2}\left(S^{1}\right) \times H^{1}\left(S^{1}\right) \times L^{2}\left(S^{1}\right)$. From now on, we consider (2.2) as a dynamical system in the $y$-variable. Though (2.2) is ill-posed as an initial-value problem, the bifurcation structure of periodic, heteroclinic, and homoclinic orbits of (2.2) can be discussed and analysed as for ordinary differential equations (ODEs), and we refer to [5, 31, 37, 38] for the relevant techniques and results.

Equation (2.2) is equivariant with respect to the $\mathbb{Z}_{2}$-actions

$$
\rho: U \longmapsto-U, \quad \tau: U(x) \longmapsto U\left(L_{x}-x\right)
$$

which correspond, respectively, to the $u \mapsto-u$ and $x \mapsto L_{x}-x$ symmetries of (1.1). We shall see in $\S 4.2$ below that the symmetry relevant for the patterns we found in our numerical explorations is the composition

$$
\kappa=\rho \tau: U(x) \longmapsto-U\left(L_{x}-x\right)
$$

of $\rho$ and $\tau$. The spatial dynamical system (2.2) is reversible with respect to the reverser

$$
\mathcal{R}:\left(U_{1}, U_{2}, U_{3}, U_{4}\right)^{t} \longmapsto\left(U_{1},-U_{2}, U_{3},-U_{4}\right)^{t},
$$

which corresponds to the reflection symmetry $y \mapsto-y$ of $(1.1)$, and the reverser $\kappa \mathcal{R}$. We say that a solution $u(\phi, y)$ is symmetric or reversible if $\mathcal{R} u=u$ or $\kappa \mathcal{R} u=u$; otherwise, we call it asymmetric. Finally, it was shown in [30] that (2.2) admits the Hamiltonian ${ }^{2}$

$$
\mathcal{H}(U)=\int_{0}^{2 L_{x}}\left[U_{2}\left(U_{4}+\left(1+\partial_{x}^{2}\right) U_{2}\right)-\frac{U_{3}^{2}}{2}+\frac{\left[\left(1+\partial_{x}^{2}\right) U_{1}\right]^{2}}{2}+\frac{\mu U_{1}^{2}}{2}-\frac{\nu U_{1}^{4}}{4}+\frac{U_{1}^{6}}{6}\right] \mathrm{d} x .
$$

Note that if $U=(u, 0,0,0)$ is an equilibrium of $(2.2)$, so that $u=u(x)$ is independent of $y$, then $\mathcal{H}(U)=\mathcal{E}(u)$, where $\mathcal{E}(u)$ is the $1 \mathrm{D}$ energy functional from (1.2) restricted to a periodic cell of length $2 L_{x}$.

After these preparations, we return to the dynamical systems interpretation of the 1D patterns and their bifurcation diagrams shown in Figures 1-2. Schematic plots of these diagrams and the underlying solutions are given in Figures 3 and 4, using now the spatial variable $y$ instead of $x$.

\footnotetext{
${ }^{1}$ The space $H^{k}\left(S^{1}\right)$ consists of all $2 L_{x}$-periodic functions whose first $k$ weak derivatives are square integrable or, equivalently, for which $\sum_{k \in \mathbb{Z}}\left(1+k^{2}\right)\left|a_{k}\right|^{2}<\infty$ where $a_{k}$ denote the associated Fourier coefficients.

${ }^{2}$ The canonical variables are $\left(q_{1}, q_{2}, p_{1}, p_{2}\right)=\left(U_{1}, U_{2},-\left(U_{4}+2\left(1+\partial_{x}^{2}\right) U_{2}\right), U_{3}\right) \in H^{3}\left(S^{1}\right) \times H^{2}\left(S^{1}\right) \times L^{2}\left(S^{1}\right) \times H^{1}\left(S^{1}\right)$ for the standard symplectic operator $\mathcal{J}$.
} 

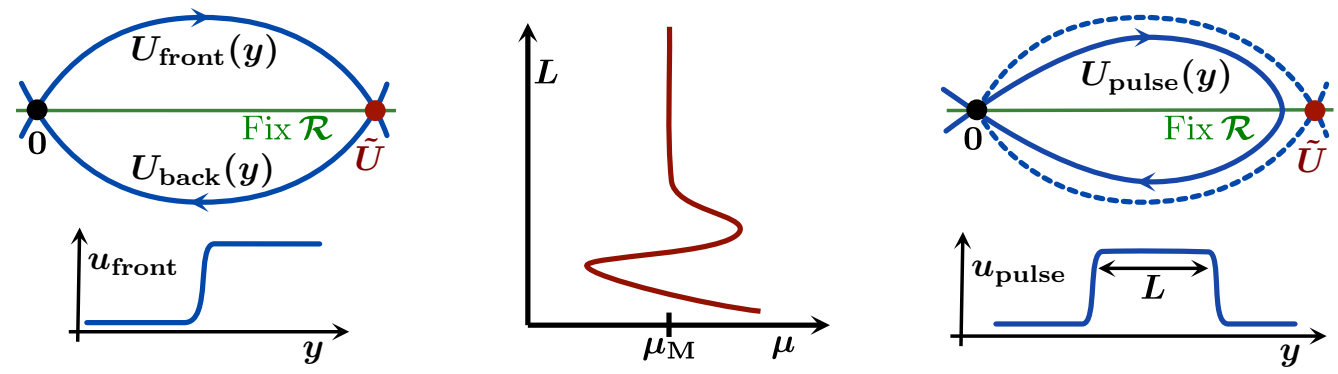

Figure 3: Non-snaking scenario: The left and right panels show respectively the initial heteroclinic cycle between the equilibria $U=0$ and $\tilde{U}$ that corresponds to fronts and their symmetric counterparts, and the bifurcating homoclinic orbit that corresponds to pulses. The centre panel illustrates the bifurcation diagram, with the $L^{2}$-norm or the width of the bifurcating pulses plotted against the bifurcation parameter $\mu$.
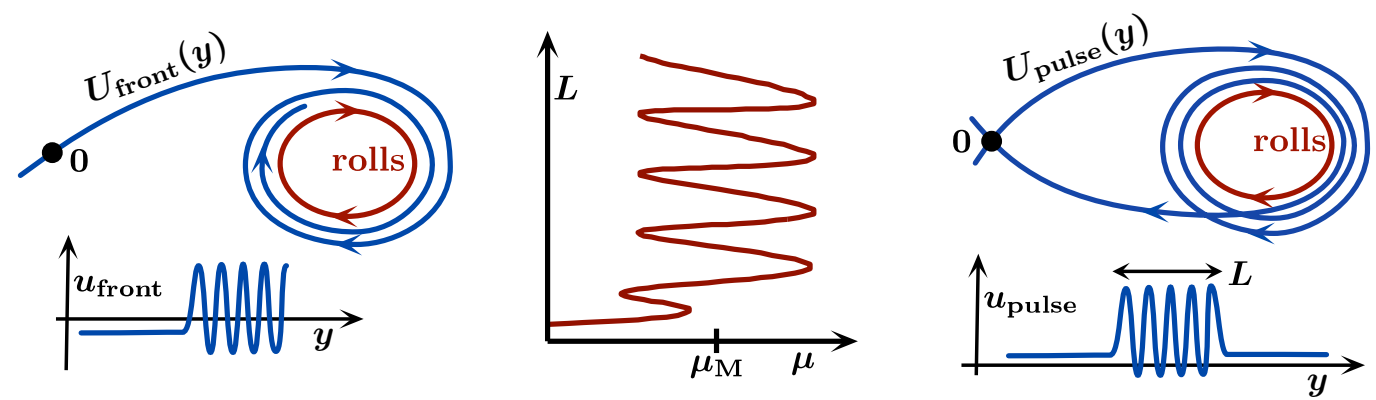

Figure 4: Snaking scenario: The left and right panels show respectively a part of the initial heteroclinic cycle between the equilibrium $U=0$ and the periodic roll pattern, and the bifurcating homoclinic orbit that corresponds to a localised roll structure. The centre panel contains the bifurcation diagram, with the $L^{2}$-norm or the width of the bifurcating pulses plotted against the bifurcation parameter $\mu$.

We begin by discussing the scenario shown in Figure 3. Assume that (2.1) admits a front $u_{\text {front }}(y)$ that connects the trivial state $u=0$ to another symmetric rest state $u=\tilde{u} \in \mathbb{R}$. Fronts of this type correspond to heteroclinic orbits $U_{\text {front }}(y)$ between the reversible equilibria $U=0$ and $\tilde{U}=(\tilde{u}, 0,0,0)$ of the dynamical system (2.2). Reversibility implies that $\mathcal{R} U_{\text {front }}(y)$ is a heteroclinic orbit between $\tilde{U}$ and 0 , and these two heteroclinic orbits therefore form a heteroclinic cycle. If the asymptotic rest states are both temporally stable, then they correspond to hyperbolic equilibria of (2.2). In particular, heteroclinic orbits are codimension one, and the fact that (2.2) is conservative implies that the cycle can exist only when the values of the Hamiltonian $\mathcal{H}$ evaluated at $U=0$ and $\tilde{U}$ coincide. Thus, the cycle can exist only when $\mathcal{H}(\tilde{U})=0$. This criterion selects a parameter $\mu=\mu_{\mathrm{M}}$, which we refer to as the Maxwell point ${ }^{3}$ of $\tilde{U}$. As shown above, $\mathcal{H}(\tilde{U})=\mathcal{E}(\tilde{u})$, and we therefore have $\mathcal{H}(\tilde{U})=\mathcal{E}(\tilde{u})=0$ at the Maxwell point of $\tilde{U}$. In particular, we can connect two different 1D solutions by an interface in the $y$-direction only if their $1 \mathrm{D}$ energies coincide. In the ODE case, it was shown in [25] that a family of symmetric homoclinic orbits to the equilibrium $U=0$ bifurcates from the heteroclinic orbit. The homoclinic orbits are parametrised by their width $L$ and exist for parameters $\mu=\mu(L)$, where $\mu(L) \rightarrow \mu_{\mathrm{M}}$ as $L \rightarrow \infty$. In particular, when plotting $\mu(L)$ against $L$, we obtain bifurcation diagrams such as the one found in Figure 2 or sketched in Figure 3. We remark that the Maxwell point for the nontrivial equilibrium shown in Figure 2 occurs at $\mu_{\mathrm{M}}=1.6367$, which gives excellent agreement with the vertical asymptote of the numerically computed bifurcation curve. Though the results in [25] are formulated for ODEs, the arguments in $[31,37,38]$ imply that they also hold for $(2.2)$.

Next, we discuss the situation shown in Figure 4, where we assume that (2.1) has a front $u_{\text {front }}(y)$ that connects the trivial state $u=0$ to a symmetric spatially periodic roll pattern $u_{\text {rolls }}(y)$. This front corresponds to a heteroclinic orbit $U_{\text {front }}(y)$ of $(2.2)$ that connects $U=0$ to a reversible periodic orbit $U_{\text {rolls }}(y)$ of the $y$-dynamical system. Heteroclinic orbits of this type can exist only when the Hamiltonian $\mathcal{H}$ vanishes along

\footnotetext{
${ }^{3}$ Since we consider localised structures, one of the two limiting rest states will be $U=0$, which justifies calling $\mu_{\mathrm{M}}$ the Maxwell point of $\tilde{U}$, and not of the pair $(0, \tilde{U})$.
} 

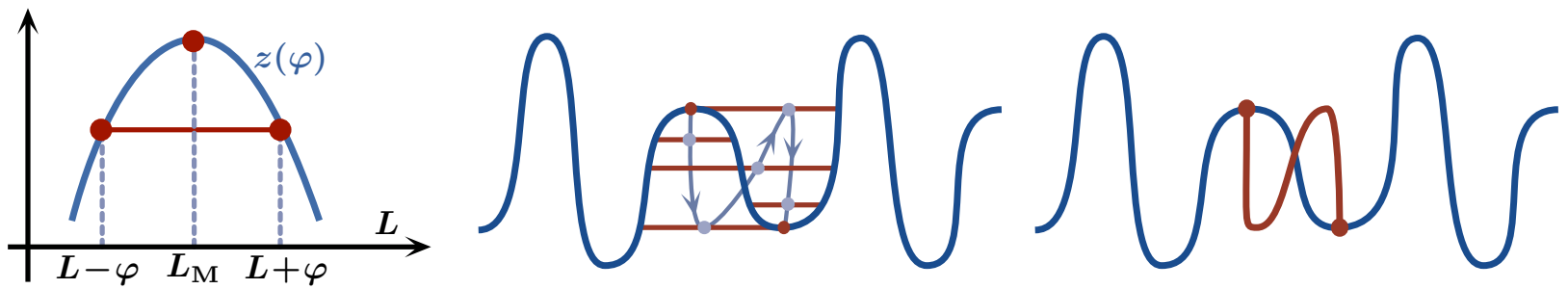

Figure 5: Solutions $(L, \varphi)$ of (2.3) are determined by the abscissas $L \pm \varphi$ of the intersection points of a fixed horizontal line segment with the graph of $z$. Continuing these solutions by moving the line segment up and down generates the entire bifurcation branch. If $\varphi$ reaches $\pi$, the branch terminates at the second snaking curve [reproduced from [5]].

the periodic orbit $U_{\text {rolls }}(y)$ so that $\mathcal{H}\left(U_{\text {rolls }}(y)\right)=0$ for one, and hence all, $y$. Periodic orbits of Hamiltonian systems come in one-parameter families that are, generically, parametrised by the value of the Hamiltonian $\mathcal{H}$. Thus, we expect that a periodic orbit $U_{\text {rolls }}(y)$ with vanishing Hamiltonian exists for $\mu$ in an open interval. Furthermore, we expect that the unstable manifold of $U=0$ intersects the stable manifold of the periodic orbit transversely inside the zero-level set of $\mathcal{H}$. Hence, heteroclinic orbits between $U=0$ and the periodic orbit $U_{\text {rolls }}(y)$ will exist over an open interval in parameter space and disappear at its end point via saddlenode bifurcations. Reversibility implies again that the heteroclinic orbit and its symmetric counterpart form a heteroclinic cycle. Homoclinic orbits that bifurcate from this cycle will correspond to localised structures that contain a long plateau where the solution resembles a spatially periodic roll pattern as sketched in Figure 4 and found in Figure 1. The results in [5] show that these homoclinic orbits will exist in the open parameter interval for which the heteroclinic cycle exists; further details of the bifurcation structure will be discussed below. We end this discussion by noting that there does not seem to be a way of predicting the existence interval of fronts. However, the variational structure of (1.1) suggests that a stationary front that connects $u=0$ to a spatially periodic pattern may exist when the energies $\mathcal{E}$ of the two asymptotic patterns are equal. Thus, if we define the Maxwell point of a periodic pattern $u_{\text {rolls }}(y)$ with $\mathcal{H}\left(U_{\text {rolls }}(y)\right)=0$ as the parameter value $\mu_{\mathrm{M}}$ for which its energy $\mathcal{E}\left(u_{\text {rolls }}\right)$ vanishes, then the heuristic argument given above indicates that the Maxwell point $\mu_{\mathrm{M}}$ might lie in the existence interval of fronts. This criterion turns out to be remarkably predictive: in Figure 1, for instance, the vertical asymptotes of the limiting fold bifurcations are $\mu=0.624$ and $\mu=0.7126$, while the Maxwell point of rolls occurs at $\mu_{\mathrm{r}}=0.6753$.

We now briefly summarise the two scenarios described above before giving more details on the second case:

Non-snaking scenario: Heteroclinic cycles between two reversible equilibria lead to branches of symmetric homoclinic orbits with vertical asymptote given by the Maxwell point, while asymmetric structures do not bifurcate [25]; see Figure 3 .

Snaking scenario: Heteroclinic cycles between an equilibrium and a reversible periodic orbit lead to two branches of symmetric homoclinic orbits that oscillate between two distinct parameter values and to branches of asymmetric homoclinic orbits [5]; see Figure 4.

In the snaking scenario, if the heteroclinic orbits exist for $\mu=z(\varphi)$, where $\varphi \in S^{1}$ is the phase of the underlying periodic orbit, then it was shown in [5] that symmetric pulses that spend time $2 L$ near the periodic orbit exist for the parameter value $\mu$ if and only if

$$
\mu=z\left(L+\varphi_{0}\right)+\mathrm{O}\left(\mathrm{e}^{-\eta L}\right), \quad L \gg 1, \quad \varphi_{0} \in\left\{0, \frac{\pi}{2}, \pi, \frac{3 \pi}{2}\right\} .
$$

Here, $\varphi_{0}=0, \pi$ correspond to even patterns that are $\mathcal{R}$-symmetric, so that $u(x, y)=u(x,-y)$, while $\varphi_{0}=\pi / 2,3 \pi / 2$ correspond to odd patterns with $\kappa \mathcal{R}$-symmetry for which $u(x, y)=-u\left(L_{x}-x,-y\right)$; the two different possible values of $\varphi_{0}$ in each of the above two cases reflect the fact that we get two patterns, namely $u$ and $\kappa u$, for each fixed reverser. Exponentially close to the folds of the snaking branch, asymmetric pulses bifurcate from the symmetric pulses. The associated bifurcation curves can be found by solving

$$
z(L+\varphi)=z(L-\varphi)
$$




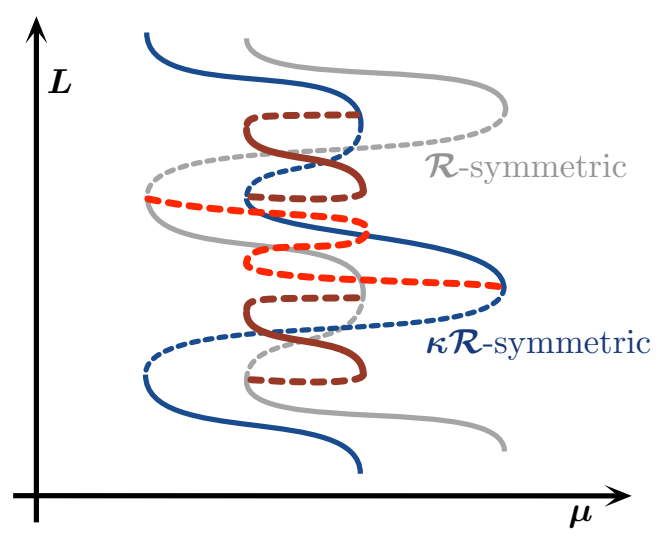

Figure 6: The two primary snaking branches correspond respectively to $\mathcal{R}$ - and $\kappa \mathcal{R}$-symmetric localised patterns. If these branches oscillate between four saddle-node asymptotes, then the analysis in [5] predicts the ladder branches of asymmetric pulses shown above, where reconnecting and cross-connecting ladders are shown in brown and red, respectively. The formal arguments in [5, §7] further predict the stability and instability of solutions along the ladder branches as indicated above by solid and dashed lines, respectively.

for $(L, \varphi)$; here $2 L$ is again the time spent near the periodic orbit, while $\varphi \in[0,2 \pi]$ measures the degree of asymmetry, with integer multiples of $\pi / 2$ corresponding to symmetric patterns. We refer to Figure 5 for a graphical method to compute the asymmetric ladder branches. As shown in [5], the branches emerging near global maxima and minima of $z$ connect the bifurcation curve of even patterns with that of odd patterns (we refer to these ladder branches as cross-connecting). In contrast, the asymmetric ladder branches that bifurcate near non-global maxima or minima of $z$ always begin and end at the same bifurcation curve (we refer to these ladders as self-connecting). Of particular interest to our discussion is the prediction of the ladder structure for snaking branches that snake between four saddle-node limits, which we show in Figure 6 .

We note that $[5, \S 7]$ asserts that the stability properties of asymmetric ladder states can be deduced formally from the stability properties of the symmetric states: if the ladder state corresponds to abscissas $L \pm \varphi$ as illustrated in Figure 5, then it should be stable if and only if the two symmetric states of widths $L \pm \varphi$, respectively, that make up the asymmetric state are both stable. If the stability of the symmetric snaking pulses is as indicated in Figure 6, then the stability properties of asymmetric ladder states should be as shown in Figure 6 . In $\S 4.2$, we will examine the validity of this prediction numerically.

Numerically, we compute snaking branches of symmetric solutions and plot $\mu$ against the squared $L^{2}$-norm $\|u\|_{2}^{2}$ of pulses to illustrate the existence region of symmetric localised patterns. Since

$$
\|u\|_{2}^{2} \propto L+p(L)+\mathrm{O}\left(\mathrm{e}^{-\eta L}\right) \quad \text { and } \quad \mu=z\left(L+\varphi_{0}\right)+\mathrm{O}\left(\mathrm{e}^{-\eta L}\right)
$$

for some $2 \pi$-periodic function $p(L)$ and some $\eta>0$, we can use the norm $\|u\|_{2}^{2}$ to predict ladder structures provided we are sufficiently high up on the snaking branch.

\section{Numerical algorithms}

In this section, we outline the numerical algorithms we use to compute localised patterns on cylinders and the plane. We focus on stationary solutions $u(x, y)$ of the Swift-Hohenberg equation (1.1) that are symmetric with respect to reflections in $x$ and, in addition, either periodic or localised in the $x$-direction. Furthermore, we consider only solutions that are localised in the $y$-direction, so that $u(x, y)$ converges to zero as $|y| \rightarrow \infty$ uniformly in $x$. Such structures $u(x, y)$ can be found as solutions to

$$
(1+\Delta)^{2} u+\mu u-\nu u^{3}+u^{5}=0, \quad(x, y) \in \Omega_{\frac{1}{2}}
$$

on the domain $\Omega_{\frac{1}{2}}=\left(0, L_{x}\right) \times\left(-L_{y}, L_{y}\right)$ with Neumann boundary conditions. Here, $L_{y} \gg 1$ and $L_{x}$ is taken to be half the period in $x$ if $u$ is periodic in $x$ or chosen to satisfy $L_{x} \gg 1$ if $u$ is localised in $x$. 

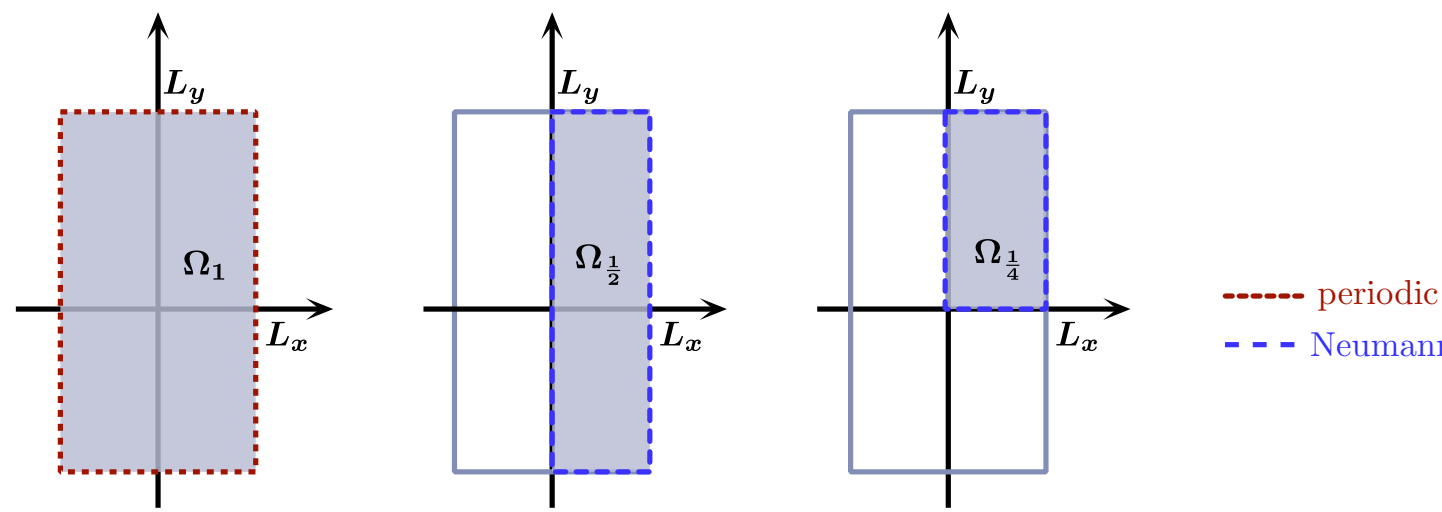

- - - Neumann

Figure 7: Computational domains used: (a) The full cylinder domain $\Omega_{1}=\left(-L_{x}, L_{x}\right) \times\left(-L_{y}, L_{y}\right)$. (b) Domain $\Omega_{\frac{1}{2}}=\left(0, L_{x}\right) \times\left(-L_{y}, L_{y}\right)$ used to compute patterns that are not symmetric in the $y$-variable. (c) Domain $\Omega_{\frac{1}{4}}=$ $\left(0, L_{x}\right) \times\left(0, L_{y}\right)$ used to compute patterns that are symmetric in the $y$-variable.

Translation invariance of the Swift-Hohenberg equation on the original unbounded domain implies that any fixed translate $u\left(x, y+y_{0}\right)$ of a stationary solution $u(x, y)$ is also a solution. Restricting $y$ to the bounded interval $\left(-L_{y}, L_{y}\right)$ with $L_{y} \gg 1$ breaks the translational symmetry. However, solutions that are localised in $y$ will interact only weakly with the boundary, and we therefore expect that each translate $u\left(x, y+y_{0}\right)$ will remain an approximate solution. In particular, the linearization about a localised structure will be close to singular, and Newton's method may not converge. To deal with this problem, we impose a phase condition that selects one specific translate from the family $u(x, y+\cdot)$ of solutions on the unbounded domain. In order to solve for this additional constraint, we exploit the fact that weak interaction with a boundary turns the formerly stationary solutions into waves that travel with a very small speed $c$ in the $y$-direction. Thus, introducing the speed $c$ as an extra parameter allows us to solve the phase constraint, and we refer to [11] for more details and a rigorous justification of this procedure. The system consisting of the phase condition and the Swift-Hohenberg equation formulated in a frame that moves with speed $c$ in the $y$-direction is given by

$$
\begin{aligned}
(1+\Delta)^{2} u+c u_{y}+\mu u-\nu u^{3}+u^{5} & =0, \quad(x, y) \in \Omega_{\frac{1}{2}} \\
\iint_{\Omega_{\frac{1}{2}}} u_{y}^{\text {old }}\left(u-u^{\text {old }}\right) \mathrm{d} x \mathrm{~d} y & =0,
\end{aligned}
$$

where $u^{\text {old }}$ is a reference solution (for instance, the initial guess or a solution computed previously for a different parameter value). The phase condition (3.3) corresponds to setting the derivative of the function $y_{0} \mapsto\left\|u\left(\cdot, \cdot+y_{0}\right)-u^{\text {old }}(\cdot, \cdot)\right\|_{2}^{2}$ at $y_{0}=0$ to zero and replacing the derivative $u_{y}$ by $u_{y}^{\text {old }}$, which is justified when $u$ is close to $u^{\text {old }}$; equation (3.3) therefore selects the approximate solution closest to $u^{\text {old }}$ in the $L^{2}$-norm. The derivative of (3.2)-(3.3) with respect to $(u, c)$ is then regular, and (3.2)-(3.3) can be solved for $(u, c)$, with $c$ approximately zero, using Newton's method.

The stability of a solution $u(x, y)$ of (3.1) can be determined by linearizing (3.1) about $u(x, y)$ and finding the rightmost eigenvalues of the resulting linear operator posed on $\Omega_{\frac{1}{2}}$ with Neumann boundary conditions (which is not equivalent to the stability on the full domain $\Omega_{1}=\left(-L_{x}, L_{x}\right) \times\left(-L_{y}, L_{y}\right)$ ).

If $u$ is reflection-symmetric in $y$, in addition to the other symmetries mentioned above, then we may compute it using the equation

$$
(1+\Delta)^{2} u+\mu u-\nu u^{3}+u^{5}=0, \quad(x, y) \in \Omega_{\frac{1}{4}}
$$

on the domain $\Omega_{\frac{1}{4}}=\left(0, L_{x}\right) \times\left(0, L_{y}\right)$ with Neumann boundary conditions. In this case, the boundary condition at $y=0$ factors out the approximate translation symmetry, and we can solve (3.4) directly using Newton's method, see [11]. Where possible, we will use this approach.

It remains to discuss how we discretise and then solve the above equations in space. We used three different programs for our computations: 
1. TRILINOS/PARACONT: To solve (3.4) on large computational domains $\Omega_{\frac{1}{4}}$, we use a 13 -point finitedifference stencil for the spatial discretisation and solve the resulting system using the continuation framework PARACONT [3, 30], a module built on top of the package TRILINOS [20]. PARACONT makes use of the parallel solver algorithms implemented in TRILINOS, which allows us to continue localised structures such as worms on quite large domains. We used PARACONT only ${ }^{4}$ for solutions with reflection symmetry in $y$ on the domain $\Omega_{\frac{1}{4}}$

2. AUto07P [18]: To continue solutions of (3.2)-(3.3), we followed the strategy outlined in [30] for a slightly different setting: we discretise the circumferential variable $x$ using a Fourier-Galerkin approximation (using only cosine functions to accommodate the Neumann conditions) and solve the resulting boundary-value problem in the remaining $y$-variable in AUTO07P. The advantages are that AUTO07P uses an adaptive mesh in the $y$-direction and runs on multiple processors; the main disadvantage is that stability computations are not possible within AUTO07P. AUTO07P's branch-switching facility failed near pitchfork bifurcations of symmetric structures. Instead, we break the $y \mapsto-y$ symmetry of the equation by adding the term $\gamma \sin (y) u$, with $0<\gamma \ll 1$, to the right-hand side of (3.2). Adding this term breaks the pitchfork bifurcation and allows us to find asymmetric branches. In our computations, we work with $n_{x}=5$ Fourier modes on the cylinder, since higher Fourier modes are found to be of order $\mathrm{O}\left(10^{-7}\right)$, and use the default values of the AUTO constants with NTST $=400$.

3. MATLAB/EPCONT: Our third implementation allows us to solve (3.1) directly, without having to add a phase condition, and to compute simultaneously the rightmost eigenvalues of the PDE linearization to test for stability. We use a modified version of the MATLAB continuation toolbox EPCONT, written by Schilder [40], which provides a general framework for continuing solutions to algebraic equations. We made two changes to EPCONT: First, we use MATLAB's Newton trust-region solver FSOLVE, which works very well for problems with singular Jacobian. Second, we projected out the approximate translation directions in each predictor step during the continuation. We discretise (3.1) using spectral differentiation matrices in the circumferential $x$-direction, see $[43, \S 3]$ for details, and finite differences in the $y$-direction. Typical mesh sizes are $n_{x}=8$ Fourier modes and $n_{y}=800$ equidistant points on the domain $(0, \pi) \times(-50,50)$. The rightmost eigenvalues of the linearization of (3.1) with Neumann or Dirichlet boundary conditions are computed in MATLAB with the sparse eigenvalue solver EIGS using the shift-invert option. The shift is initialised using an a priori bound for the spectrum and then updated with the rightmost eigenvalue during continuation. We typically computed the 10 rightmost eigenvalues. Stability was also checked using an initial value problem solver.

\section{Localised stripe patterns on cylinders}

In this section, we consider the equation

$$
(1+\Delta)^{2} u+\mu u-\nu u^{3}+u^{5}=0, \quad(x, y) \in \Omega_{\frac{1}{2}}
$$

on the domain $\left(0, L_{x}\right) \times\left(-L_{y}, L_{y}\right)$ with Neumann boundary conditions. Unless stated otherwise, we take $\nu=2$ throughout this section. We focus on patterns that are localised in the $y$-direction and both symmetric and periodic in the $x$-direction with period $2 L_{x} \approx 2 \pi$. Bifurcation diagrams are visualised by plotting the parameter $\mu$ against the squared $L^{2}$-norm $\|u\|_{L^{2}}^{2}$ of the underlying pattern. Patterns $u(x, y)$ are visualised by colour plots, with $x$ plotted horizontally and $y$ vertically, where the values of $u(x, y)$ are represented by different colours as indicated in the colour bars in Figures 8-9 below. In particular, red and blue colours correspond respectively to positive and negative values of $u$. To better visualise patterns, we duplicate their profiles several times in the periodic $x$-direction in all colour plots. Finally, unless stated otherwise, the

\footnotetext{
${ }^{4}$ PARACONT now also works with phase conditions but we implemented this feature only at a later stage.
} 

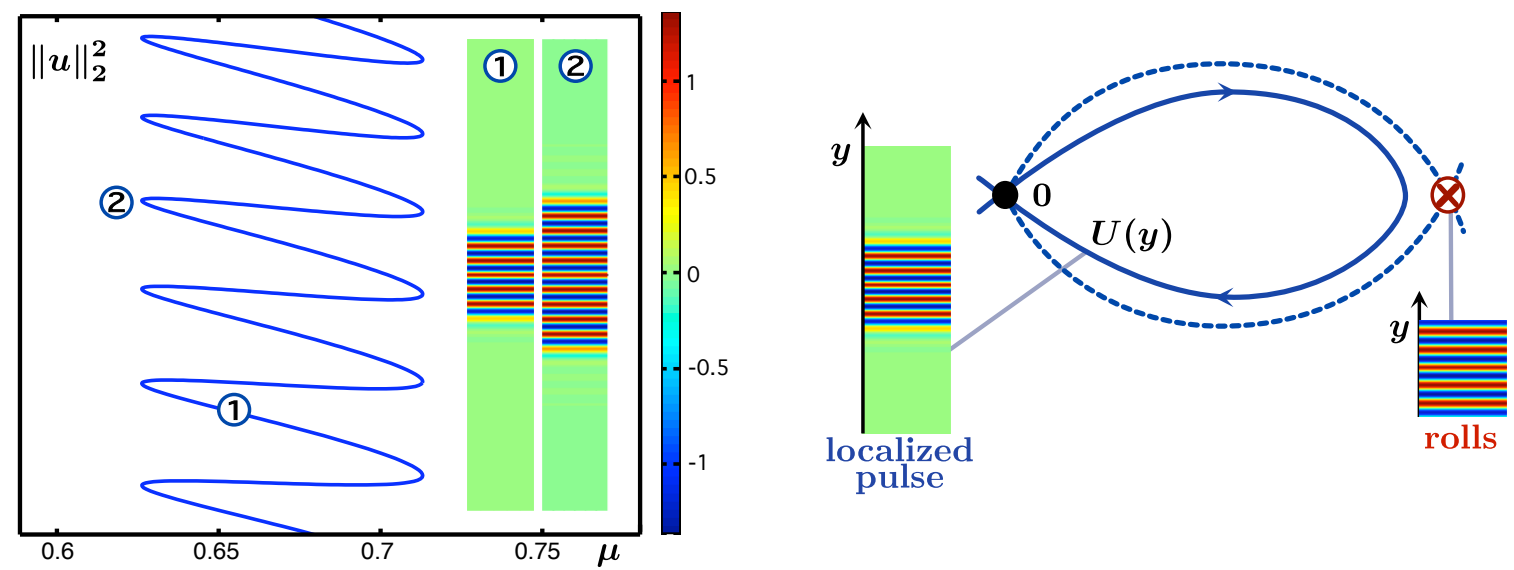

Figure 8: The left panel contains the bifurcation diagram for snaking planar localised rolls for $\nu=2$. The associated spatial-dynamics interpretation for the $y$-evolution is shown in the right panel. The roll pattern shown in the right panel corresponds to a periodic orbit in the y-dynamics: throughout this paper, we indicate equilibria of the y-dynamics by filled circles and periodic orbits (in an appropriate Poincaré section) by circled crosses.
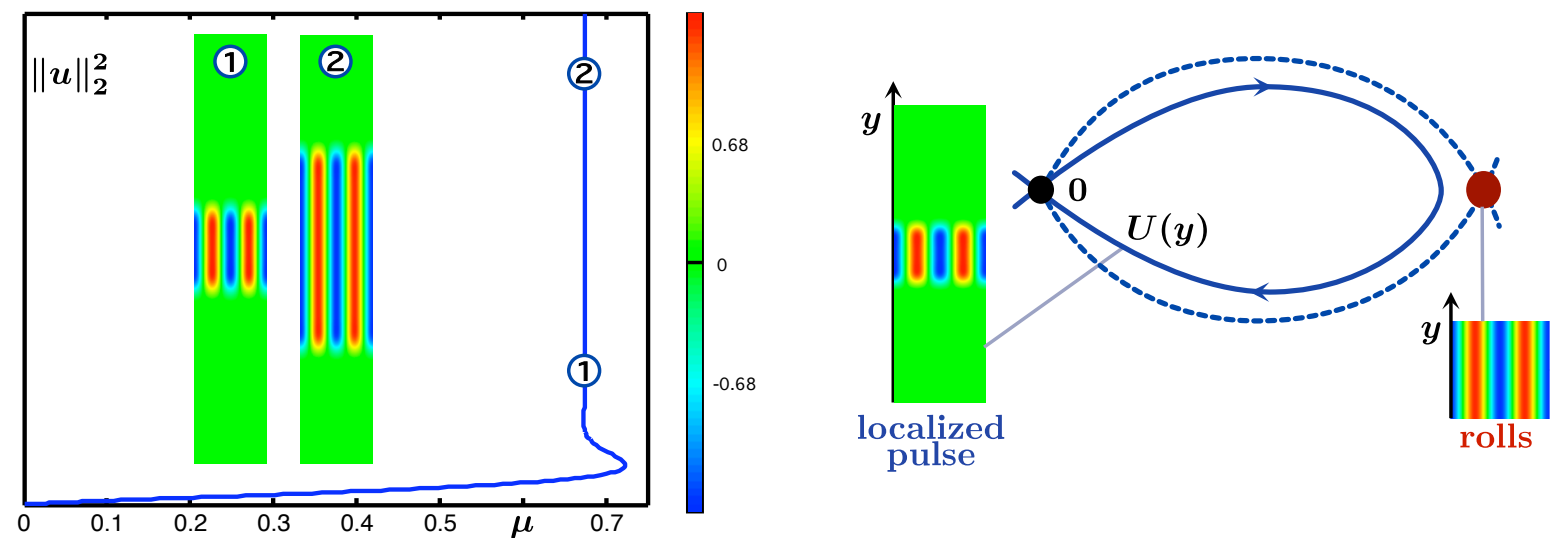

Figure 9: Non-snaking localised rolls for $\nu=2$ are shown in the left panel, while the associated spatial-dynamics interpretation for their y-evolution is shown in the right panel. The roll pattern shown in the right panel corresponds to an equilibrium of the $y$-dynamics. Recall that equilibria are indicated by filled circles.

stability of patterns that are periodic in the $x$-direction with period $2 L_{x}$ is computed on the interval $\left(0, L_{x}\right)$ with Neumann boundary conditions.

\subsection{Planar localised rolls}

The one-dimensional localised structures found in Figure 1 can be viewed as planar localised rolls. Figure 8 shows their bifurcation diagram (which is, of course, identical to those of localised 1D roll patterns) and their interpretation in terms of the spatial dynamical system (2.2) in the evolution variable $y$. The rolls in the interior of the localised structure are periodic in the vertical $y$-variable, and localised planar rolls can therefore be viewed as $\mathcal{R}$-reversible homoclinic orbits that arise near a heteroclinic cycle from the equilibrium $U=0$ to a periodic solution that corresponds to the $y$-periodic roll pattern: this explains why snaking occurs. We remark that the localised rolls shown in Figure 8 are found to be alternately stable and unstable.

On the other hand, instead of orienting rolls parallel to the interface with the trivial state as in the case of Figure 8, we may seek localised roll structures for which the rolls are oriented perpendicular to the interface with the trivial background state. Figure 9 indicates that such structures indeed exist but that they do not snake. Non-snaking of these structures is consistent with their spatial-dynamics interpretation. As indicated in Figure 9, roll patterns that are comprised of rolls that are oriented parallel to the $y$-direction are periodic in $x$ but constant in $y$ and correspond therefore to equilibria of the $y$-dynamical system. Thus, the 

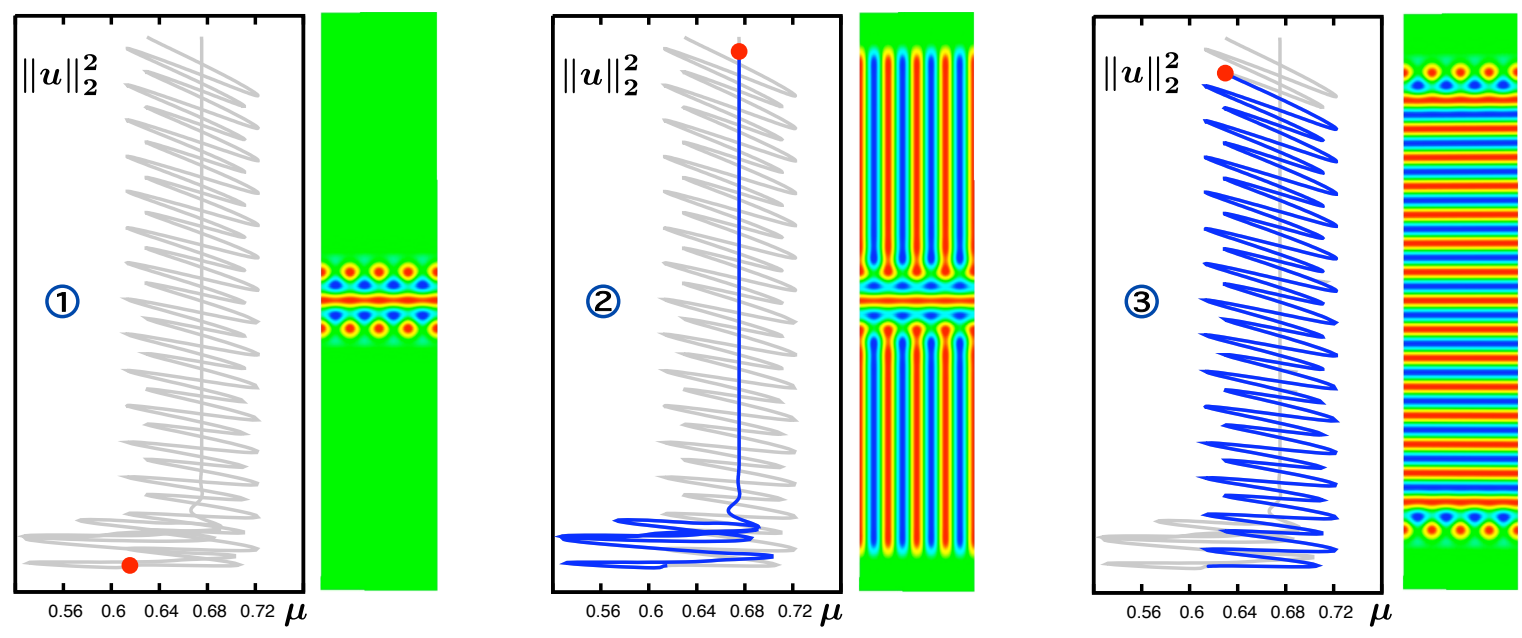

Figure 10: Panel 1 contains the bifurcation diagram of an almost planar stripe pattern: the pattern profile corresponding to the red dot is shown to the right. Decreasing $\mu$ leads to the non-snaking branch shown in panel 2. Increasing $\mu$, on the other hand, leads to the snaking branch shown in panel 3: an interior roll section is developed which grows as we move along the branch. The family of solution profiles along the entire bifurcation curve can be viewed in the accompanying movie. Solutions are computed for $\nu=2$ with algorithm 1 on $\Omega_{\frac{1}{4}}$ with $L_{x}=12.6, L_{y}=62.8, n_{x}=33$, and $n_{y}=129$.

corresponding localised rolls can be interpreted as homoclinic orbits in the $y$-evolution that bifurcate from a heteroclinic cycle that involves only equilibria, namely the trivial state $U=0$ and the parallel roll pattern. In particular, the discussion in $\S 2$ implies that snaking should not occur. Direct numerical simulations indicate that the localised rolls in Figure 9 are again alternately stable and unstable between saddle-node bifurcations.

\subsection{Almost planar localised rolls}

The spatial-dynamics interpretation of the localised roll patterns discussed in Figures 8 and 9 allowed us to predict quite easily whether they should snake or not. In this section, we consider localised patterns that can exhibit both snaking and non-snaking behaviour depending on the parameter direction in which we continue them. In particular, the initial shape of the pattern is not sufficient to predict snaking or non-snaking.

Our starting point is the pattern shown in the left panel of Figure 10, which was found in [9] via direct numerical simulations for $\nu=2$. We use algorithm 1 to continue this pattern in the parameter $\mu$ and refer to the accompanying movie for a complementary visualization of the patterns we found. Upon continuing initially in the direction of decreasing $\mu$, we observe the non-snaking scenario. As shown in the centre panel of Figure 10 and in more detail in Figure 11, vertical rolls are formed at both ends of the pattern that then grow in length as we move along the branch. The bifurcation branch itself approaches the vertical asymptote $\mu_{\mathrm{r}}=0.6753$, which corresponds to the Maxwell point of 1D rolls. As indicated in Figure 12, this behaviour can be explained as follows by spatial dynamics in the $y$-variable. An almost planar stripe pattern corresponds to a reversible homoclinic orbit that connects the trivial state to itself but that also stays for long times $y$ near the equilibrium that corresponds to the vertical $1 \mathrm{D}$ roll structure. It is then natural to postulate the existence of a heteroclinic network in the $y$-dynamics that consists of a heteroclinic cycle that connects $U=0$ to the equilibrium corresponding to vertical 1D rolls and a homoclinic orbit that connects the latter equilibrium to itself. The homoclinic orbit is $\mathcal{R}$-reversible and therefore robust. The heteroclinic orbits forming the heteroclinic cycle, on the other hand, will have codimension one as they can exist only at the Maxwell point. Since the heteroclinic network involves only equilibria and no periodic orbits when interpreted in terms of the $y$-dynamical system, we are in the non-snaking case.

We now return to Figure 10 and discuss the right panel, which we obtained when we continued the almost planar stripe pattern from the left panel initially in the direction of increasing $\mu$. The spatial-dynamics 


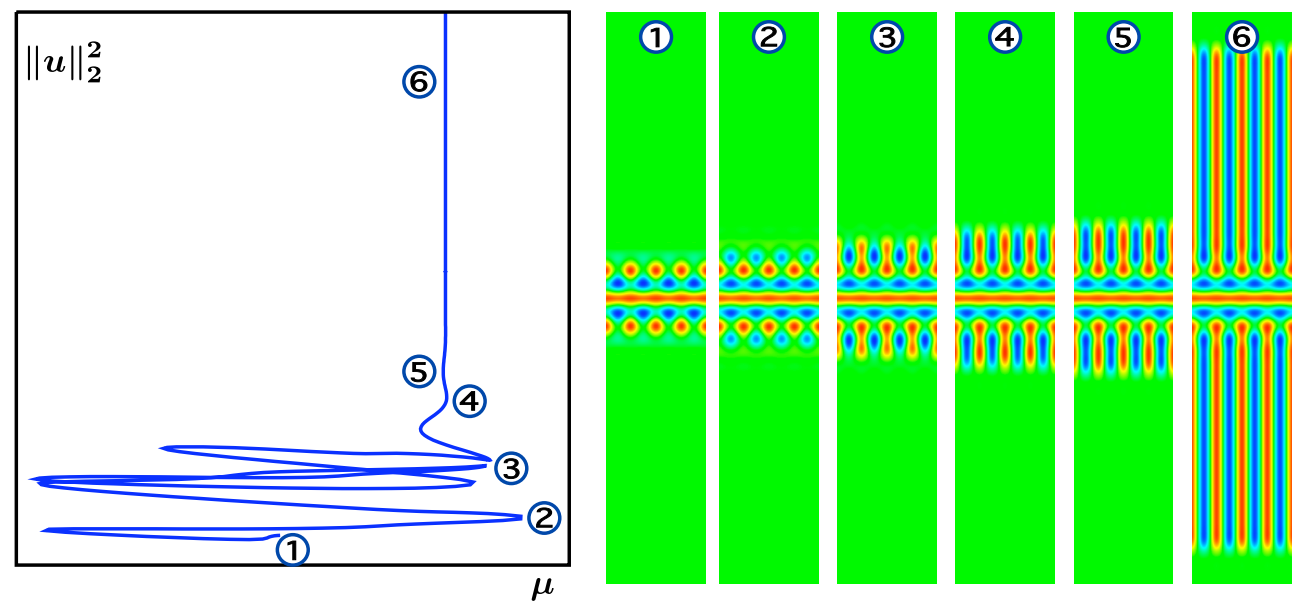

Figure 11: The non-snaking branch of the bifurcation diagram from panel 2 in Figure 10 is displayed in more detail. As we move along the branch, the solution develops blue and red spots along the interface. These red spots subsequently develop into vertical stripes, which progressively cover the entire domain. The vertical asymptote occurs at the Maxwell point $\mu_{\mathrm{r}}=0.6753$ of the $1 D$ rolls.
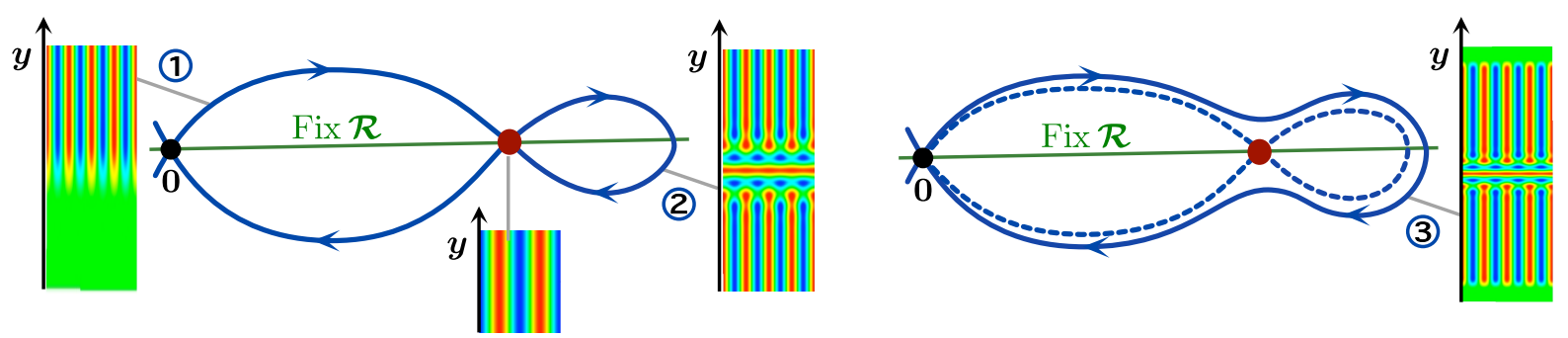

Figure 12: We illustrate the spatial-dynamics interpretation of the y-dynamics of almost planar stripes along the non-snaking branch. We can interpret the almost planar stripe pattern shown in the right panel as a homoclinic orbit to $U=0$ that bifurcates from the heteroclinic network shown in the left panel. The heteroclinic network consists of a codimension-one heteroclinic cycle between $U=0$ and vertical $1 D$ rolls, which exists only at the Maxwell point of $1 D$ rolls, and a robust reversible homoclinic orbit to vertical $1 D$ rolls. Note that vertical $1 D$ rolls are equilibria in the $y$-dynamics.
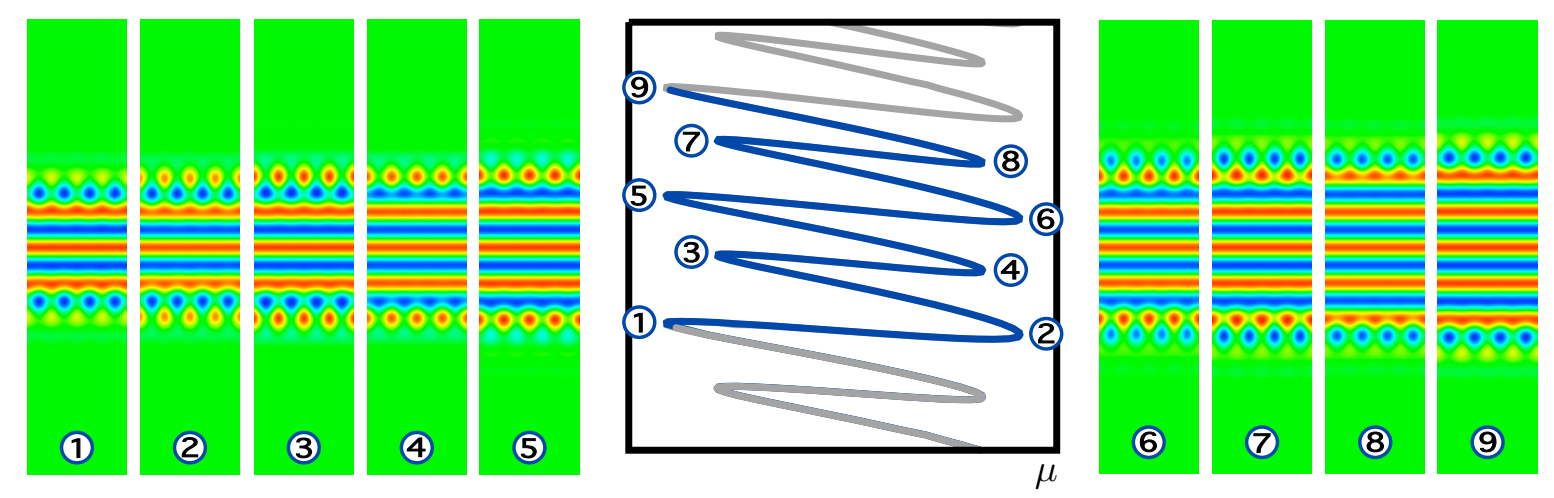

Figure 13: In the centre, we show a section of the snaking branch from the right panel of Figure 10. As we move along the branch, the pattern grows horizontal stripes via a sequence of nine saddle nodes as shown in panels (1)-(9). For the pattern shown in panel (1), the interface between rolls and the trivial state is made up of blue spots. As we move up on the branch through panels (2)-(5), red spots are added to the interface, whilst the blue spots merge to form the first half of a new roll. Panels (6)-(9) show the development of new blue spots along the interface, whilst the red spots merge to complete the formation of the new roll seen in panel (9). Inspecting panels (1) and (5), we find that the interface regions, plotted here over four full periods in $x$ so that $x \in\left(0,8 L_{x}\right)$, are related by the symmetry operator $\kappa$, which corresponds to multiplication of the pattern by -1 and reflecting it in $x$ across $x=L_{x}$. 

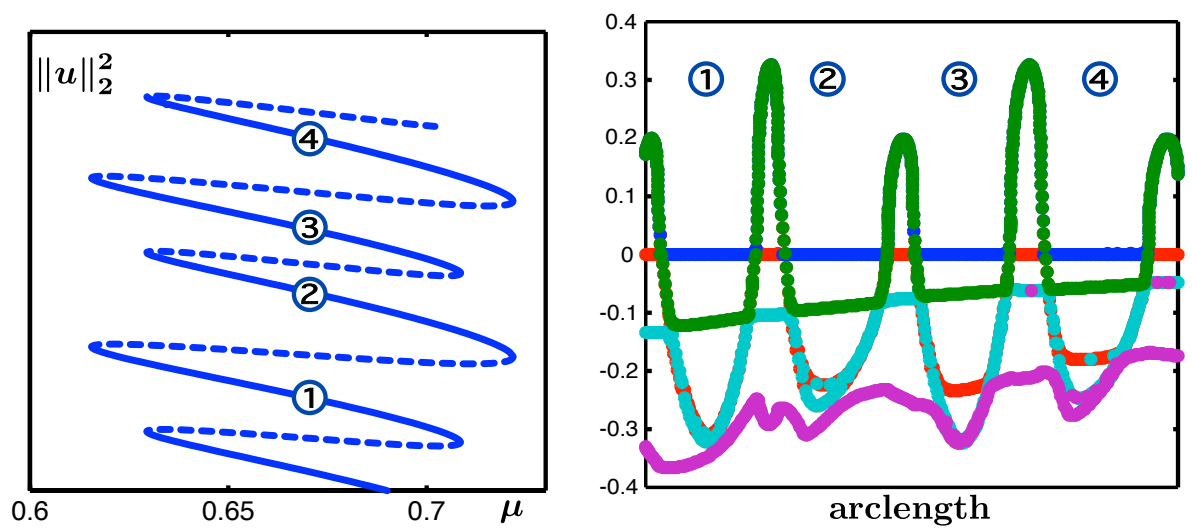

Figure 14: The stability of the $\mathcal{R}$-symmetric almost planar stripes from Figure 13 with respect to even perturbations is indicated in the left panel by solid (stable) and dashed (unstable) lines. On the unstable branches, there are two unstable eigenvalues: one unstable eigenvalue is associated with the saddle-node bifurcation, while the other one is due to a pitchfork instability that breaks the $\mathcal{R}$-symmetry. The right panel shows the eigenvalues of the linearised problem plotted along the branch. Computations are done using algorithm 3 with $n_{x}=8, n_{y}=800$, and $L_{y}=50$.
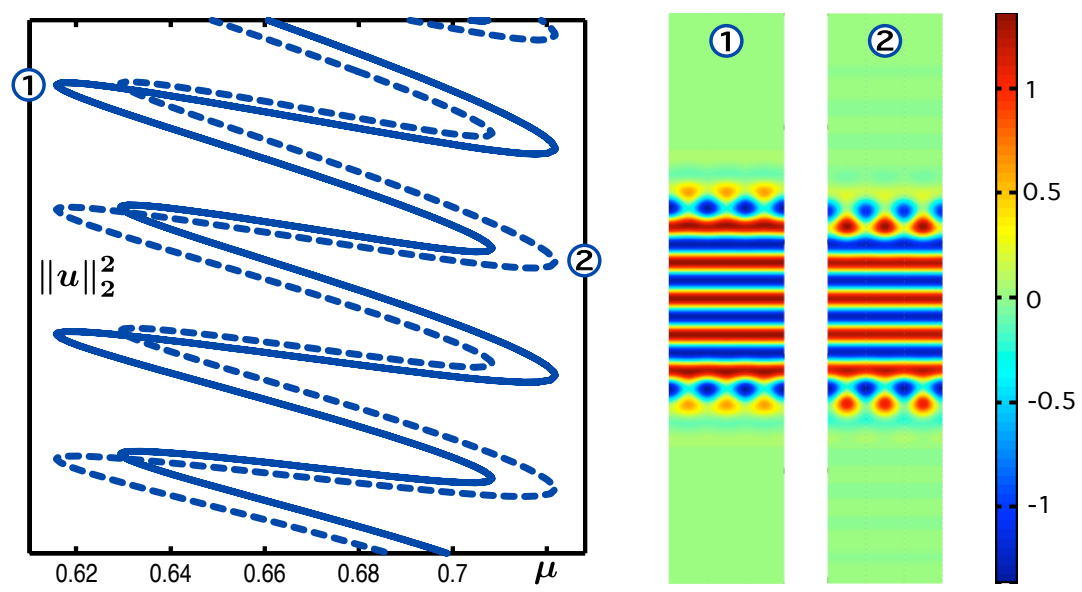

Figure 15: The solid branch corresponds to almost planar stripe patterns with $\mathcal{R}$-symmetry, while the dashed branch corresponds to solutions with $\kappa \mathcal{R}$-symmetry. The saddle-node limits are $\mu=0.6157,0.6293,0.7084,0.7218$, and the Maxwell point of $1 D$ rolls occurs at $\mu_{\mathrm{r}}=0.6753$. This branch is computed with algorithm 2 with $L_{y}=100$ and $\mathrm{NTST}=200$.

interpretation of the pattern shown in the right panel of Figure 10 is the same as that shown in Figure 8 for planar stripe pattern, which explains the snaking behaviour visible in Figure 10. Interestingly, the saddle nodes along the snaking branch are aligned along four vertical asymptotes instead of the usual two vertical limits. The details of the profile changes that occur at these saddle-node bifurcations are shown and explained in Figure 13. Note that all patterns are reversible under the reverser $\mathcal{R}$ that acts through $y \mapsto-y$. Moving up from one saddle node to the next, say from panel (1) to panel (5) in Figure 13, we see that the interface regions of the two patterns are related by the symmetry operator $\kappa$ that acts through $u(x, y) \mapsto-u\left(L_{x}-x, y\right)$. Thus, from the results in [5] that we discussed in $\S 2$, we expect the complete bifurcation diagram to be as shown in Figure 6. In particular, there should be a second distinct branch of patterns with $\kappa \mathcal{R}$-symmetry, whose bifurcation curve is shifted vertically in the $\|u\|_{L^{2}}^{2}$-direction by half of the curve's vertical period. Furthermore, various ladder branches corresponding to asymmetric patterns should exist. Before discussing these structures in detail, we mention that our spectral computations indicate that the patterns from Figure 13 are alternately stable and unstable with respect to perturbations on $\Omega_{\frac{1}{2}}$ with Neumann boundary conditions; see Figure 14. Thus, the sketch given in Figure 6 correctly reflects the stability properties along the primary snaking curve of $\mathcal{R}$-symmetric patterns.

Our goal is now to verify, via numerical computations, that the overall bifurcation structure of the almost 


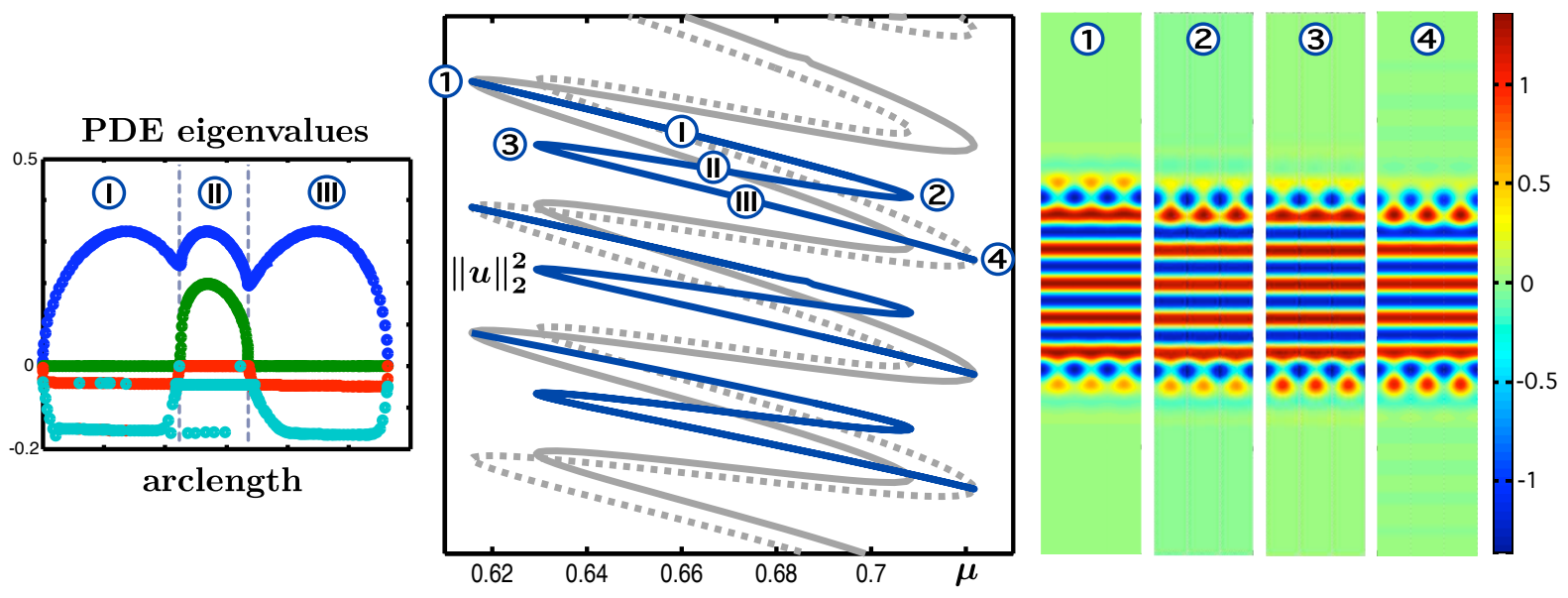

Figure 16: Shown are the cross-connecting ladder branches of asymmetric patterns that connect the branch of $\mathcal{R}$ symmetric patterns from Figure 15 to the branch of $\kappa \mathcal{R}$-symmetric patterns. The four rightmost PDE eigenvalues of the asymmetric states along the ladder branches are shown in the left panel: all solutions are unstable. All ladder branches are computed with algorithm 3 using $n_{x}=4, n_{y}=800$, and $L_{y}=50$.
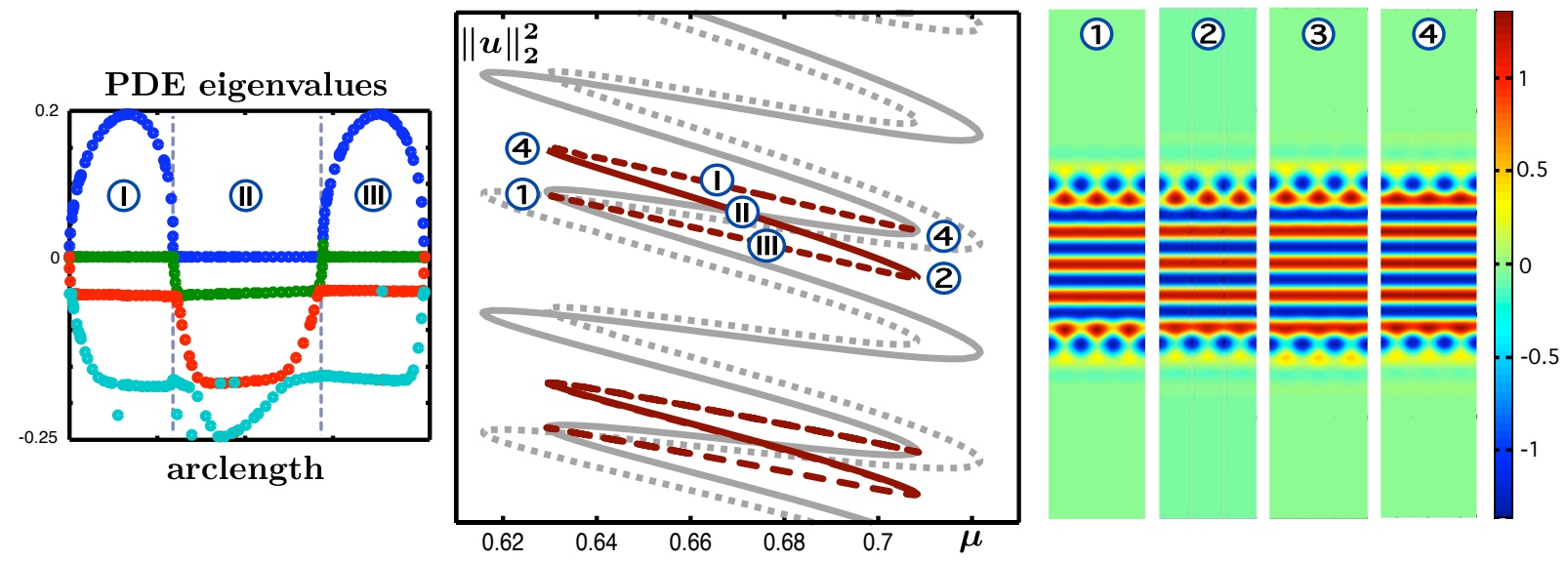

Figure 17: Shown are the reconnecting ladder branches of asymmetric patterns that begin and end at the branch of $\mathcal{R}$-symmetric patterns from Figure 15. The four rightmost PDE eigenvalues of the asymmetric states along a ladder branch are shown in the left panel, indicating that these patterns are stable along the middle parts of each ladder branch. The computational details are as in Figure 16.

planar stripe patterns, including their stability properties, is exactly as predicted in Figure 6. As already mentioned, the almost planar stripe patterns presented in Figure 13 are $\mathcal{R}$-symmetric so that $u(x, y)=$ $u(x,-y)$ for all $(x, y)$. The patterns with $\kappa \mathcal{R}$-symmetry, whose existence we conjectured above, would satisfy $u(x, y)=-u\left(L_{x}-x,-y\right)$ for all $(x, y)$, that is, they should not change when simultaneously reflecting in $y$, reflecting in $x$ across the mid-period point, and multiplying the pattern by -1 . In Figure 15, we present numerical computations of these anticipated $\kappa \mathcal{R}$-symmetric almost planar stripe patterns that exist along a snaking branch that is shifted vertically as conjectured.

Next, we computed the ladder branches of asymmetric structures that are predicted by the analysis in [5] and sketched in Figure 6. The asymmetric patterns bifurcate via pitchfork bifurcations from the symmetric patterns. These pitchforks occur exponentially close in $L$ to the fold bifurcation. Furthermore, the results in [5] show that the ladder branches that emerge near the inner folds connect back to the same branch of symmetric patterns, while the ladder branches that emanate from the outermost folds along the branch of $\mathcal{R}$-symmetric structures connect to the branch of $\kappa \mathcal{R}$-symmetric structures, and vice versa; we shall refer to the former branches as reconnecting and to the latter branches as cross-connecting. The numerical continuation results shown in Figures 16 and 17 confirm these findings. We also computed the rightmost eigenvalues of the PDE linearisation about the asymmetric stripe patterns and find that these patterns 

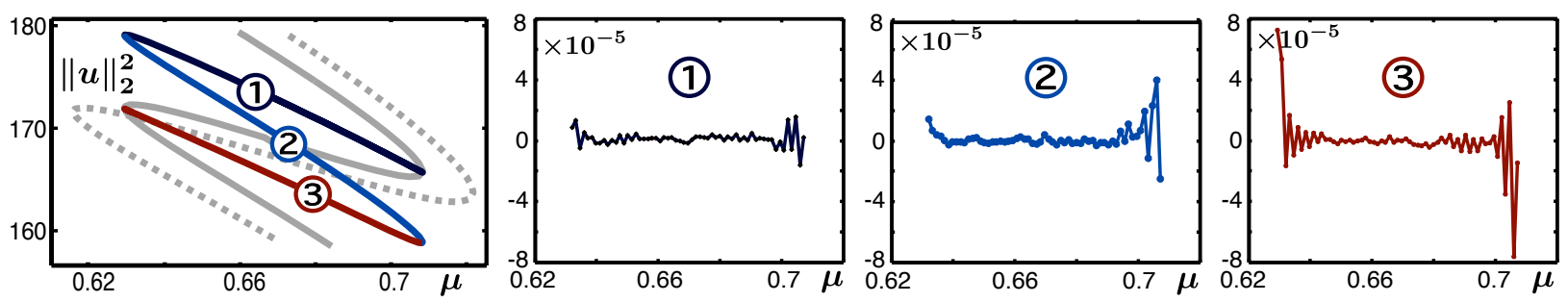

Figure 18: We compare the numerically computed ladder branches and the curves predicted from the snaking branch using the equation $z(L+\varphi)=z(L-\varphi)$. The larger errors at the end points of the ladder branches are due to interpolation errors that arise since we used an equidistant interpolation mesh which leads to the Runge phenomenon. The interpolation is done with 60 points along each branch.
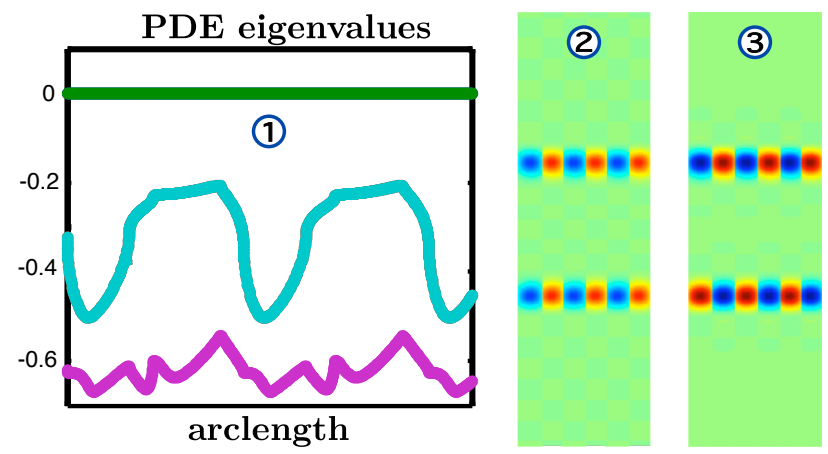

Figure 19: The four rightmost Dirichlet eigenvalues of the patterns shown in Figure 13 are plotted in the left panel against the arclength along the branch. Two of these eigenvalues are very close to zero, and the resulting eigenfunctions are shown in the centre and right panels. One of the small eigenvalues sits at zero and is enforced by the translation symmetry in the periodic $x$-direction, while the other one reflects the fact that the almost planar stripe patterns can be thought of as bound states (see main text for details). Computations were performed with algorithm 3 using $n_{x}=4$, $n_{y}=800$, and $L_{y}=50$.

are unstable along the cross-connecting branches and stable along the middle parts of the reconnecting branches. To test the analytical prediction for the asymmetric states, we plot in Figure 18 the difference of the numerically computed solution branches and the curves computed from the symmetric branch via the equation $z(L+\varphi)=z(L-\varphi)$, for which we find excellent agreement.

The spectral computations we presented so far address linear stability with respect to perturbations that satisfy Neumann boundary conditions at $x=0$ and $x=L_{x}$ (and therefore on the boundary of the computational domain $\Omega_{\frac{1}{2}}$; see Figure 7 ). To address spectral stability on the full cylindrical domain $\Omega_{1}$ with periodic boundary conditions in $x$, it suffices to compute the spectrum on $\Omega_{\frac{1}{2}}$ with Dirichlet boundary conditions at $x=0$ and $x=L_{x}$. In Figure 19, we plot the Dirichlet spectrum of the almost planar stripe patterns given in Figure 13, but we note that we obtained similar results for the other symmetric and asymmetric patterns that we computed above. Figure 19 shows that these patterns have two Dirichlet eigenvalues near zero. One of these eigenvalues arises due to the translational symmetry in $x$ and should therefore sit exactly at the origin. The other eigenvalue arises due to the bound-state nature of our solutions. Indeed, the central part of each almost planar stripe pattern consists of horizontal roll patterns that are approximately constant in the $x$-direction. Thus, if we shift the solution in the upper and lower half of the domain against each other in the $x$-direction, the resulting pattern is still an approximate solution. Among this one-parameter family of approximate patterns, which is parametrised by the relative shift in the $x$-direction, there are two exact solutions, one of which is $\mathcal{R}$-symmetric, while the other has $\tau \mathcal{R}$-symmetry. We expect that one of these two symmetric patterns is stable under periodic boundary conditions, while the other one is unstable in the direction of the shift $x$. 

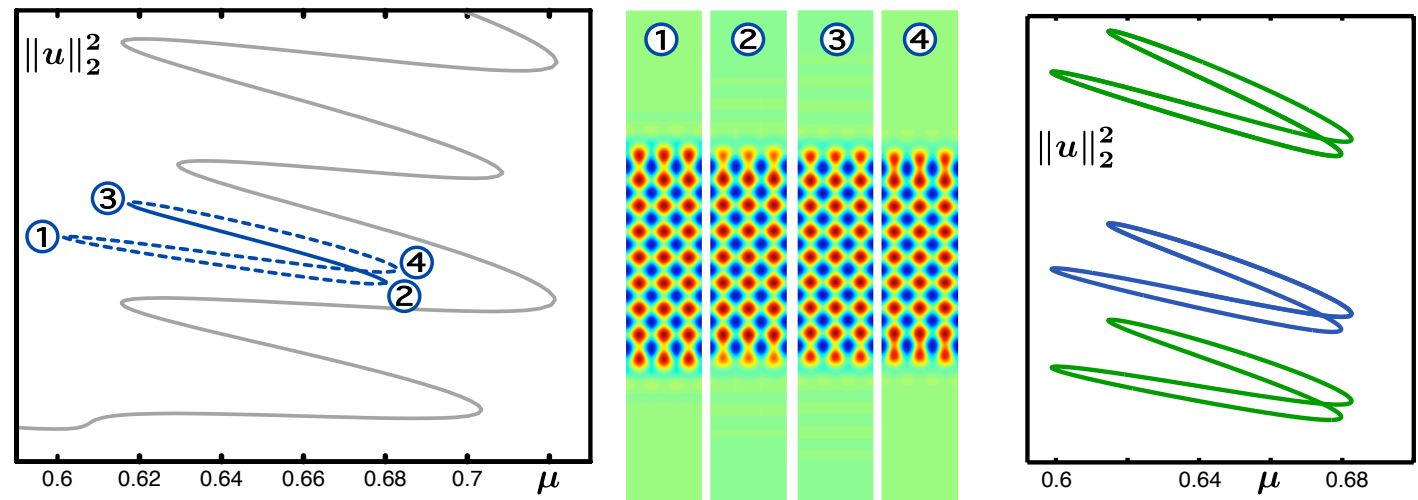

Figure 20: The left and right panels contain isolas of localised square patterns for $\nu=2$. In the left panel, stable and unstable solutions along the isola are indicated by solid and dashed curves, respectively; the isola from the left panel is drawn in blue in the right panel. Algorithm 2 was used with $y \in[0,200]$ and $\mathrm{NTST}=400$.
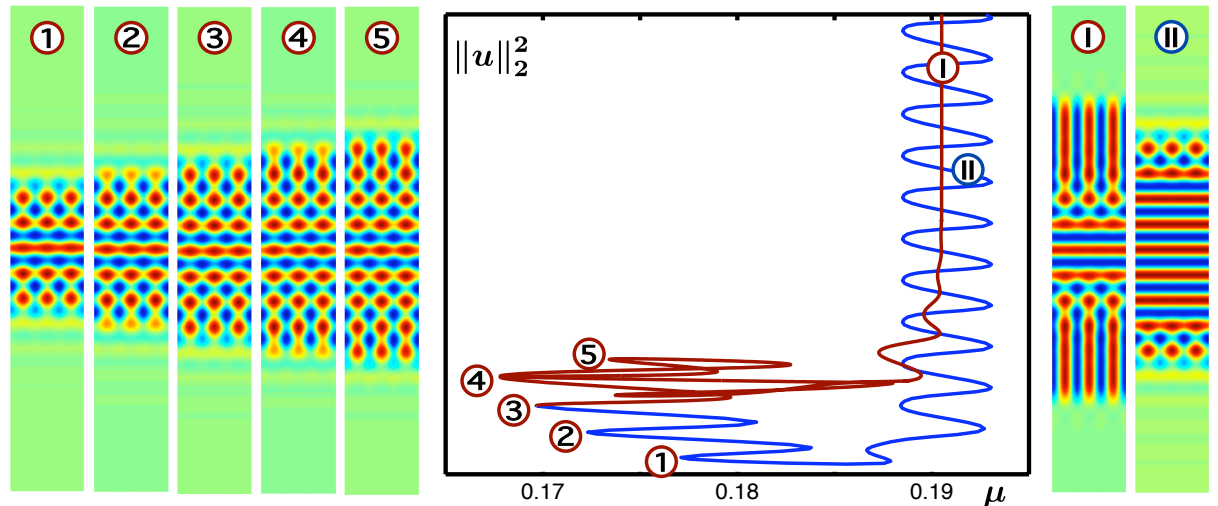

Figure 21: The bifurcation diagram of almost planar stripe patterns is shown for $\nu=1.06247$. The Maxwell points of rolls and squares occur at $\mu_{\mathrm{r}}=0.1905$ and $\mu_{\mathrm{s}}=0.1716$, respectively. The branch is computed with algorithm 2 with $y \in[0,200]$ and $\mathrm{NTST}=300$.

\subsection{From localised stripe to square patterns}

For the almost planar stripe patterns that we discussed in the previous section, we observed the growth of square cells along the interface between rolls and the trivial state that then merged to form new rolls. This observation led us to examine localised square patterns on the cylinder. Figure 20 contains continuation results for localised square patterns that we found near the Maxwell point $\mu_{\mathrm{s}}=0.609$ of domain-filling square patterns. The pattern profiles from panels (1)-(4) show that new squares are grown at the interface with the trivial state. The bifurcation curve is an isola, though, and snaking does not occur. It appears as if the snaking of the pattern is inhibited by the relative proximity of the roll structures: panel (4) indicates that the localised square pattern tries to grow vertically oriented rolls. Note that the localised square patterns shown in Figure 20 seem to be stable along part of the bifurcation curve, which indicates that domain-covering square patterns are stable in this parameter region. This is surprising since domain-covering square patterns are known to be unstable at onset in the cubic-quintic Swift-Hohenberg equation [14].

To further explore the interaction between stripe and square patterns, we lowered the value of $\nu$ to 1.06247 and again continued localised almost planar stripe patterns in $\mu$. The results, shown in Figure 21, indicate that the bifurcation structure is qualitatively similar to that shown in Figure 10 for $\nu=2$. Starting from the pattern shown in panel (1) of Figure 21, one side of the branch approaches a vertical asymptote given by the Maxwell point of 1D rolls, while the other side of the branch snakes. Along the non-snaking branch, the pattern grows four rows of squares as in panels (2)-(5) before eventually growing vertically oriented rolls as in panel (I). Along the snaking branch, an interior plateau of horizontally oriented rolls develops as shown in panel (II). There are a few interesting differences both in terms of the shape of the profiles and the details of 

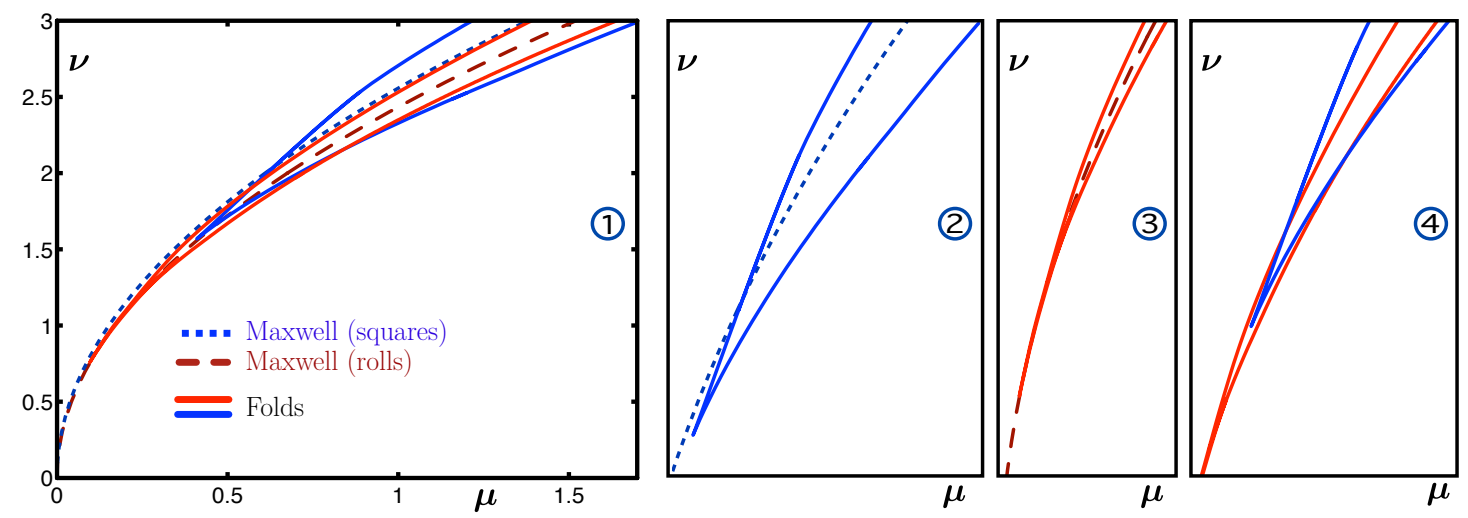

Figure 22: The left panel shows four saddle-node bifurcation curves of the almost planar localised stripe patterns from Figure 13 in the $(\mu, \nu)$-plane, together with the Maxwell curves associated with domain-filling roll and square patterns. The left-most and right-interior folds in Figure 13 are plotted in red, while the right-most and left-interior folds in Figure 13 are shown in blue. The remaining three panels contain enlarged portions of panel (1).
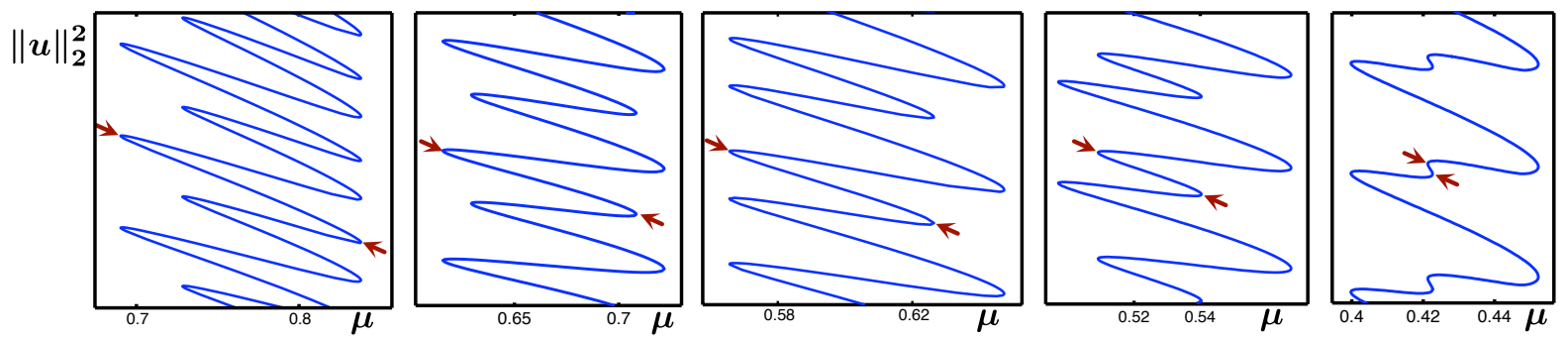

Figure 23: The bifurcation diagrams of almost planar stripe patterns are shown for $\nu=2.153,2,1.895,1.806,1.599$ from left to right. The arrows indicate which of the folds correspond to each other upon varying $\nu$.

the bifurcation curves when comparing Figures 10 and 21. In the latter figure, many more rows of squares are grown, indicating that the Maxwell points of squares and rolls are much closer to each other so that these patterns can bind more strongly to each other. Second, there are only two saddle-node asymptotes along the snaking branch shown in Figure 21 compared with four limits in Figure 10. In particular, for $\nu=1.06247$, we expect to find only horizontal ladder states of asymmetric localised stripe patterns similar to those shown in Figure 1, compared with the more complicated bifurcation structures visible in Figures 16-17 for $\nu=2$.

To determine which of the two left and two right folds for $\nu=2$ collide and disappear when we decrease $\nu$, we continued these folds in two parameters. Figure 22 contains the resulting two-parameter bifurcation diagram with the four fold curves of the almost planar stripe patterns from Figure 13 and the Maxwell curves associated with stripes and squares. We find that the left-most and right-interior folds of the stripe patterns shown in Figure 13 collide in a cusp bifurcation at $(\mu, \nu)=(0.401,1.57)$, while the right-most and left-interior folds from Figure 13 disappear in a cusp at $\nu=0.758$. These results explain why the snaking curve in Figure 22 for $\nu=1.06247$ involves only two vertical asymptotes. To illustrate these results further, we plot in Figure 23 the bifurcations curves of almost planar stripe patterns for various values of $\nu$, while tracking the location of all folds along the snaking diagram. We see again how the left-most fold collides with the innermost right fold for $\nu \approx 1.57$. Note that different ladder branches for asymmetric patterns will collide and reorganise themselves near the parameter values corresponding to panels (1), (3), and (5) in Figure 23, where the folds change their relative position in the $\mu$-direction: the theory developed in [5] can be used quite straightforwardly to analyse the resulting global bifurcations but we shall not present these details here.

Note that the Maxwell curves for stripes and squares shown in Figure 22 emerge both from the codimensiontwo point $(\mu, \nu)=0$. The proximity of the Maxwell curves for squares and rolls suggests that snaking in the cubic-quintic Swift-Hohenberg equation may be very intricate. Indeed, the numerical computations in $[8,9]$ indicate that the snaking behaviour of localised patterns in $1 \mathrm{D}$ is very complicated in the presence of 

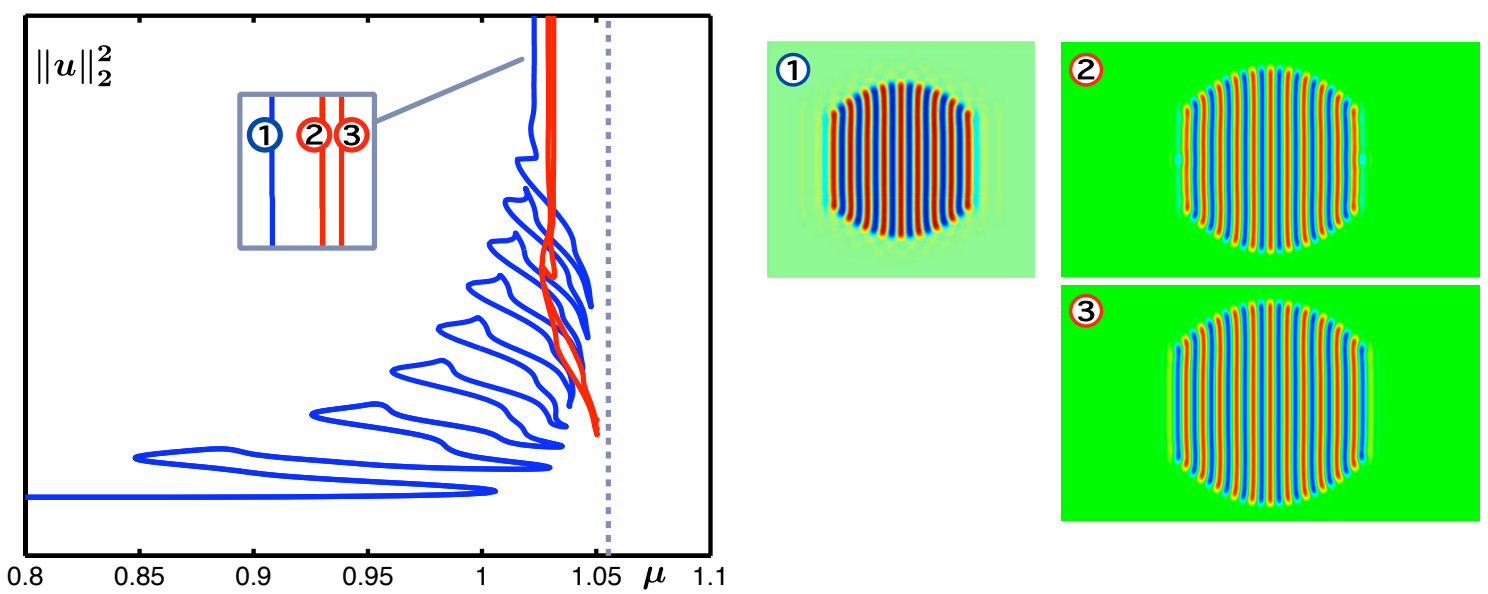

Figure 24: The left panel contains two disjoint branches of fully localised planar stripe patterns and the Maxwell point $\mu_{\mathrm{r}}=1.0560$ of $1 D$ rolls shown as the vertical dashed line. The blue curve, which corresponds to the worm patches with 9 rolls shown in panel (1), approaches the vertical asymptote $\mu=1.0228$. For comparison, the Maxwell point of $1 D$ localised roll patterns comprised of 9 rolls is given by $\mu=1.0023$. The red curve corresponds to the worm patches with 11 rolls shown in panels (2)-(3): its limiting vertical asymptotes are $\mu=1.0290$ and $\mu=1.0314$. The Maxwell points of localised $1 D$ roll patterns with 11 rolls are $\mu=1.0285$ and $\mu=1.0313$. The red curve has been rescaled linearly in the vertical direction to fit into the same graph. The computations were done for $\nu=2.50081$ with $L_{x}=90, L_{y}=90.38$, and $n_{x}=n_{y}=220$.

multiple Maxwell points.

\section{$5 \quad$ Fully localised planar stripe patterns}

In this section, we investigate the parameter dependence of fully localised planar stripe patterns, which we refer to as worms by analogy with similar but time-dependent structures first observed in electroconvection in nematic liquid crystals [17, 24]. In the planar Swift-Hohenberg equation, time-independent states of this type were first found in [36] using direct numerical simulation. In both systems, these states are located in the subcritical regime [6,36]. Our goal is to investigate via continuation methods whether worm structures in the Swift-Hohenberg equation snake or not. We found stationary worms initially by continuing radial spots from which worms bifurcate in symmetry-breaking bifurcations. This is similar to the case of fully localised planar hexagon patterns that were discussed in [30] for the quadratic-cubic Swift-Hohenberg equation: the hexagon patches also bifurcated from radial spots and were found to snake in a complicated fashion that is not yet fully understood. All computations in this section are carried out using algorithm 1 for large values of $L_{x}$.

Figure 24 contains two disjoint branches of fully localised stripe patterns for $\nu=2.50081$. The branch shown in blue emanates from a branch of radial spots and begins to snake, but eventually ceases to snake and instead approaches a single vertical asymptote. During the snaking phase, the worm profiles acquire an additional pair of vertically aligned rolls at each fold bifurcation, thus making the patterns shown in panel (1) of Figure 24 wider in the horizontal $x$-direction as we move up along the branch. As snaking ceases and the branch approaches its vertical asymptote, the number of rolls, and therefore the horizontal width, of the localised worm pattern stays constant; instead, the rolls grow along the vertical $y$-direction, thus making the pattern more elongated in the vertical direction, which leads to the observed increase of its $L^{2}$-norm. Figure 24 contains a second branch, shown in red, which starts and ends at infinite $L^{2}$-norm and can therefore be thought of as an isola of localised worm patterns. The worm profiles consist of 11 rolls whose vertical length changes along the branch to generate the change in $L^{2}$-norm visible in Figure 24 . As shown in the inset, this second branch exhibits two different vertical asymptotes.

Surprisingly, the three asymptotes of the branches plotted in Figure 24 are relatively far away from the 

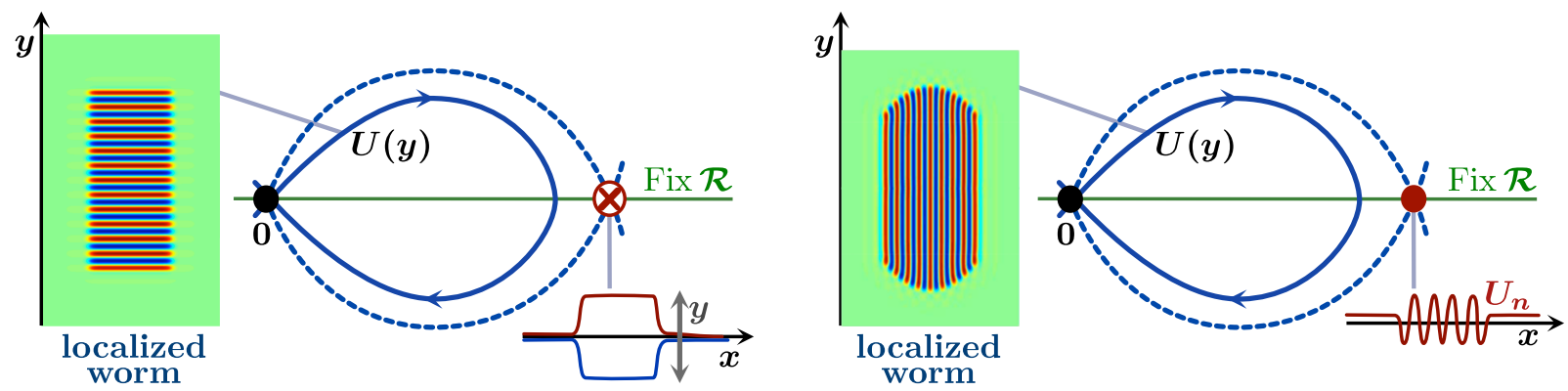

Figure 25: The left panel shows a fully localised stripe pattern with rolls that are aligned horizontally. If only the vertical length of this pattern (and therefore its $L^{2}$-norm) increases during continuation, while its horizontal width stays constant, then the stripe patches can be interpreted as homoclinic orbits of the $y$-dynamical system, which bifurcate from a heteroclinic cycle between the equilibrium $U=0$ (indicated by a solid circle) and the $y$-periodic orbit (indicated by a circled cross) that corresponds to the horizontally aligned rolls that are localised in the $x$-direction. This scenario is expected to lead to snaking. The right panel shows a worm patch that consists of $n$ vertical rolls. If the number of rolls stays constant during continuation, and the patterns grow only in the vertical y-direction, then we can interpret these patterns as homoclinic orbits for the $y$-dynamics that connect the trivial state to itself and stay for long times near the equilibrium that consists of a localised $1 D$ roll pattern $U_{n}(x)$ comprised of $n$ rolls. These homoclinic orbits may arise from a heteroclinic cycle that connects the equilibria $U=0$ and $U=U_{n}$ : this cycle has codimension one, and we expect to encounter the non-snaking case.
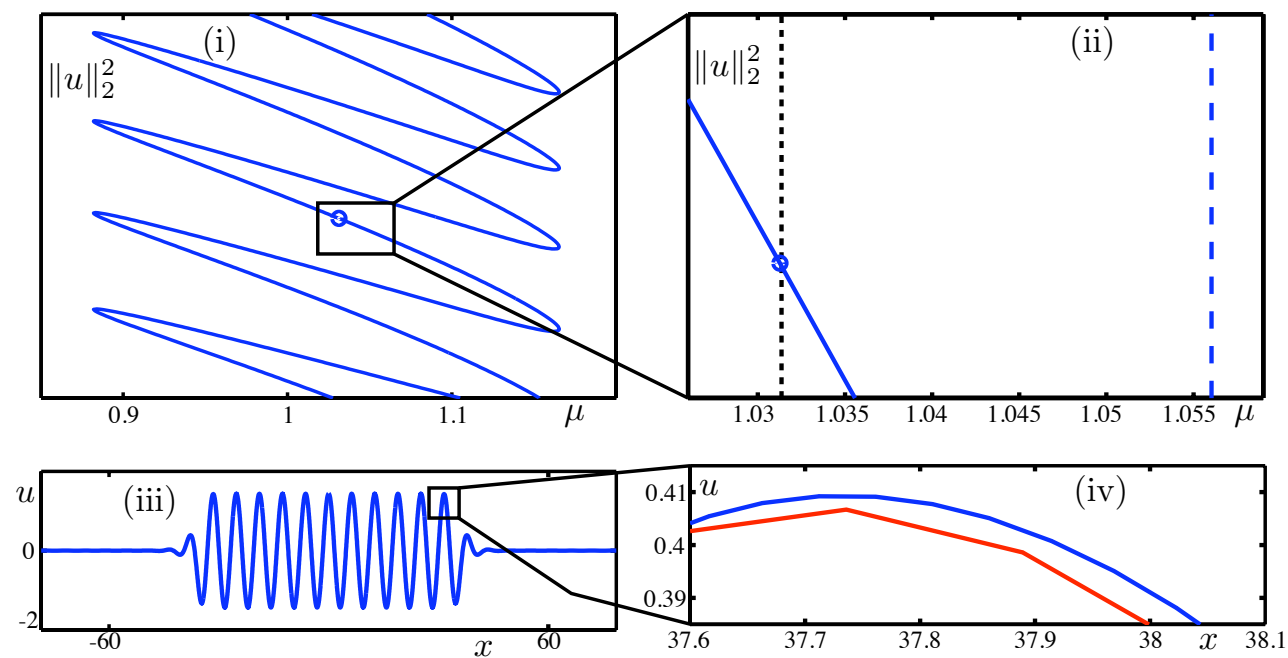

Figure 26: Panel (i) shows the bifurcation diagram of localised $1 D$ rolls at $\nu=2.50081$. The blue circle at $\mu=1.0313$ denotes the location of the $1 D$ pulse with $\mathcal{E}=0$ that consists of 11 rolls: its profile is shown in panel (iii). Panel (ii) shows a segment of the bifurcation curve near this $1 D$ pulse together with the Maxwell point $\mu=1.0560$ of $1 D$ rolls shown as a vertical dashed line and the vertical asymptote $\mu=1.0314$ of the fully localised worm patch comprised of 11 rolls from panel (3) in Figure 24 as a vertical dotted line. In panel (iv), we compare the pulse profile (in blue) and the transverse worm profile along the $x$-direction for fixed $y$ (in red).

Maxwell point of domain-filling 1D rolls, and we now put forward an explanation of this discrepancy using spatial-dynamics arguments. Figure 25 contains spatial-dynamics interpretations of the initial snaking period and the eventual non-snaking regime in the left and right panels, respectively, that are similar to those for the almost planar stripe patterns that we discussed in the previous section. The key difference between worms and planar stripe patterns is that the heteroclinic cycles for the $y$-dynamical system that describe worms do not involve domain-filling $1 \mathrm{D}$ rolls as in the case of planar stripe patterns. Instead, the limiting equilibria are localised $1 \mathrm{D}$ rolls $u_{n}(x)$ that consist of a finite number of $1 \mathrm{D}$ rolls, given by the number $n$ of vertical rolls that make up the localised worm patch. These heteroclinic cycles should exist only at the Maxwell point of the localised 1D roll patterns $u_{n}(x)$, where $\mathcal{H}\left(u_{n}\right)$ vanishes. In the caption of Figure 24, we compare the Maxwell points of the 1D pulses that consist of 9 and 11 rolls with the asymptotes of worm 


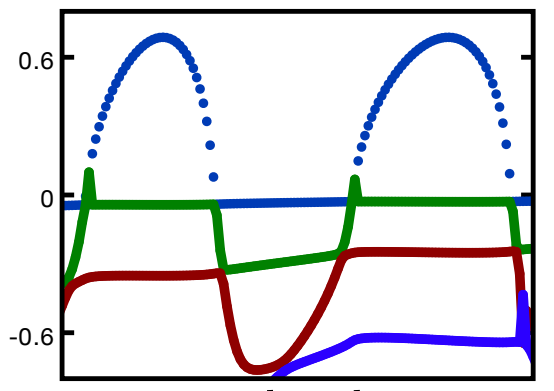

arclength

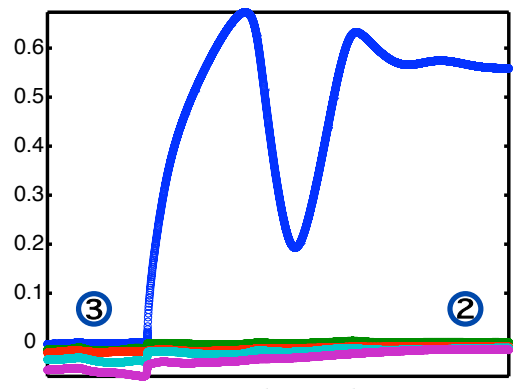

arclength

Figure 27: The left and right panels contains the rightmost PDE eigenvalues along the snaking curve of localised rolls from Figure 26 and along the red branch of fully localised planar stripe patterns with labels (2) and (3) from Figure 24 , respectively, at $\nu=2.50081$. The eigenvalues are parametrised by the arclength along the underlying solution branches.
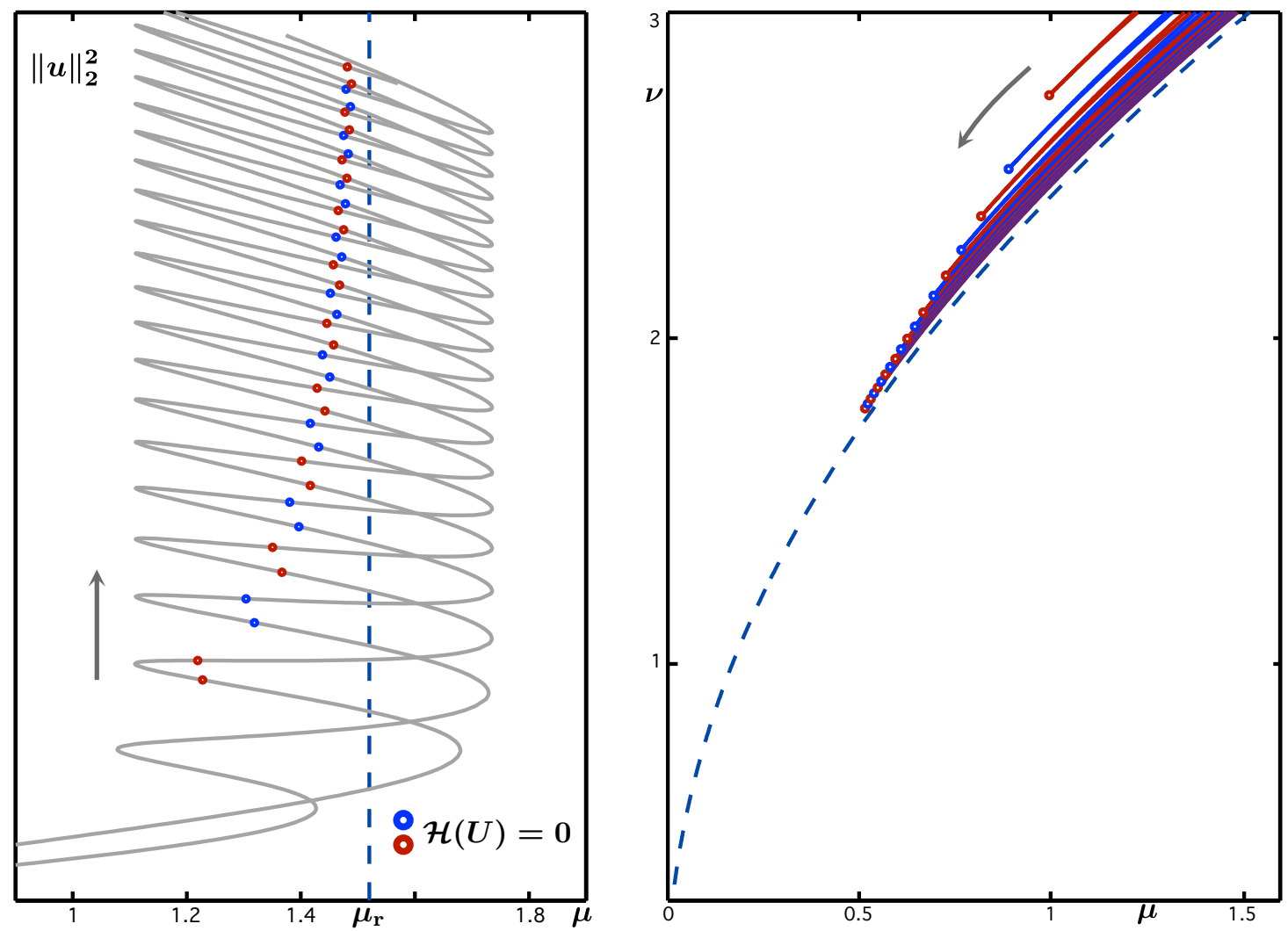

Figure 28: The left panel contains the two snaking branches of localised $1 D$ pulses for $\nu=3$. Pulses with vanishing Hamiltonian $\mathcal{H}$ are labelled with circles, and we see that the corresponding values of $\mu$ tend to the Maxwell point of $1 D$ rolls, indicated by the vertical dashed line, as the pulse width tends to infinity. The two lowest red circles correspond to pulses with 2 rolls, while the pulses at the two topmost red circles consist of 11 rolls; two rolls are added at each left fold bifurcation. Continuing $1 D$ pulses with vanishing Hamiltonian for different pulse widths in the $(\mu, \nu)$-plane leads to the diagram shown in the right panel, where the dashed curve is the Maxwell curve of $1 D$ rolls.

patches with 9 and 11 rolls and find excellent agreement. Figure 26 contains a more detailed comparison of the worm with 11 rolls and the associated 1D pulse. We remark that there are two 1D pulses with vanishing Hamiltonian $\mathcal{H}$ for each given number of rolls. These two pulses lie on the same snaking branch but are separated by a single fold bifurcation. Thus, one of them is stable, while the other one is unstable. Inspecting the red branch in Figure 24 that corresponds to worms with 11 rolls and noting that this branch exhibits two different asymptotes given by the two 1D pulses with vanishing Hamiltonian that have 11 rolls, we therefore expect that worms are stable along one part of the branch and unstable along the other part: Figure 27 contains a comparison of the rightmost PDE eigenvalues of 1D pulses and worms with 11 rolls along their 


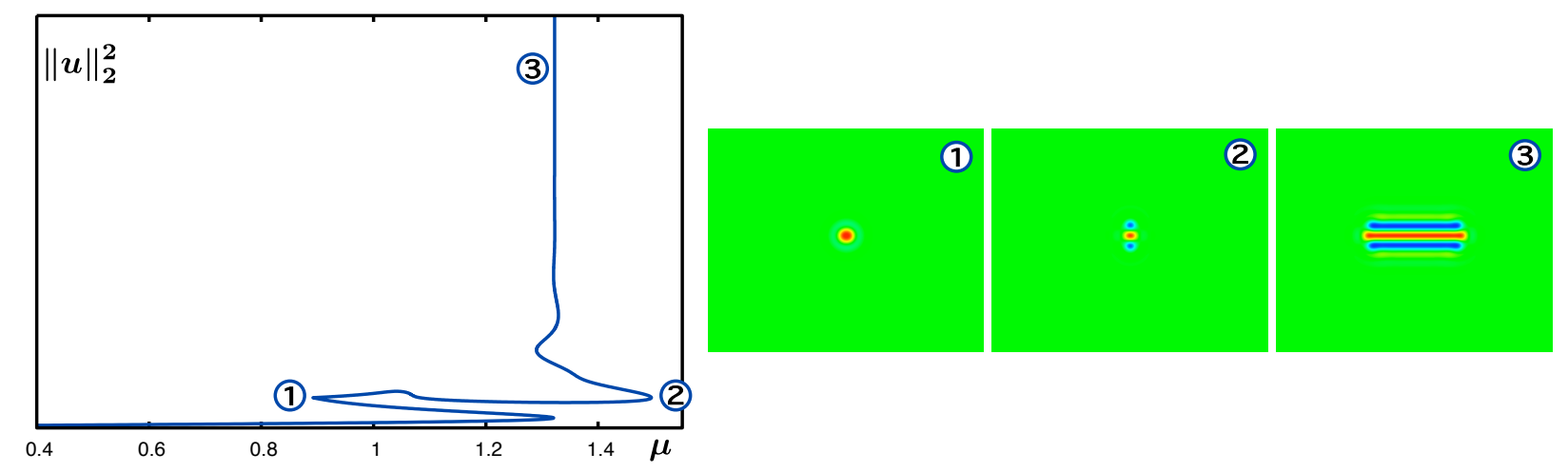

Figure 29: A branch of fully localised planar stripe patterns that consist of one roll is shown in the left panel for $\nu=3$. As we move up on the branch towards label (3), the horizontal width of the stripe pattern increases. The computations were done with $L_{x}=90, L_{y}=90.38$, and $n_{x}=n_{y}=220$.

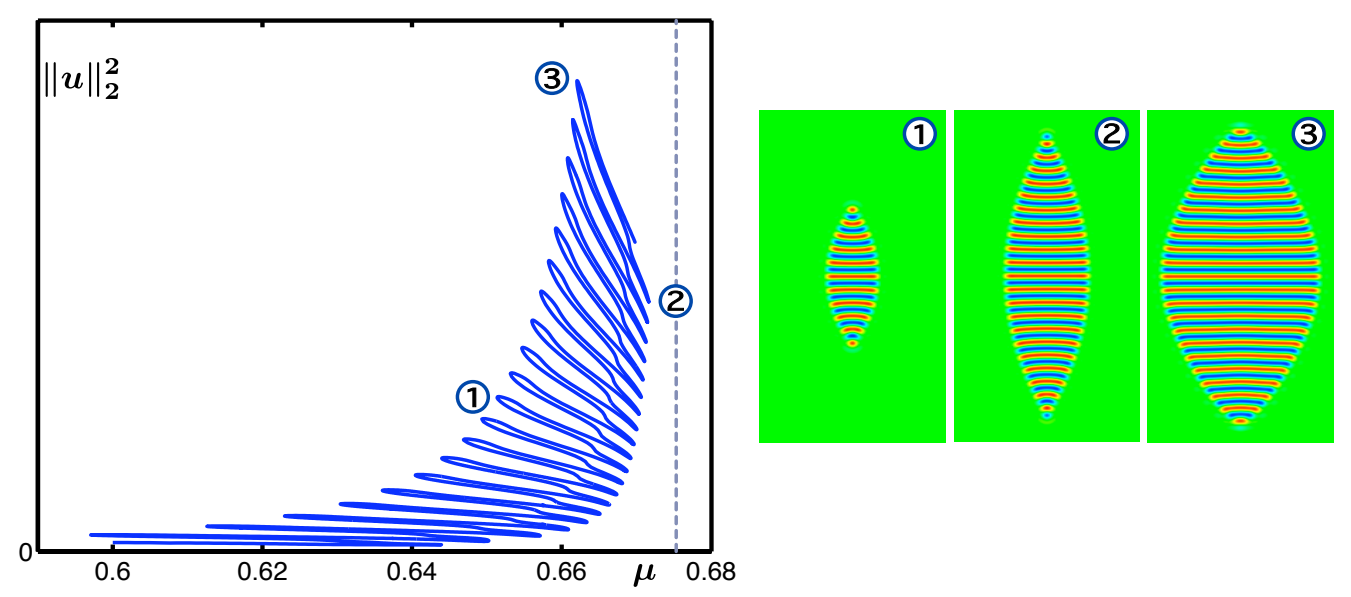

Figure 30: A branch of fully localised planar stripe patterns at $\nu=2$ is shown in the left panel. The Maxwell point of $1 D$ rolls that occurs at $\mu=0.6753$ is shown as a vertical dashed line. The computations were done with $L_{x}=43.9823$, $L_{y}=78.5398, n_{x}=129$, and $n_{y}=513$.

respective branches that confirms this expectation.

The results presented so far indicate that branches of fully localised stripe patterns with $n$ interior rolls should approach vertical asymptotes given by 1D pulses with vanishing Hamiltonian that consist of $n$ rolls. In the right panel of Figure 28, we plot the branches of $1 \mathrm{D}$ pulses with vanishing Hamiltonian in the $(\mu, \nu)$ plane for values of $n$ between 2 and 11 rolls. As we decrease $\nu$, the two branches corresponding to the stable and unstable 1D pulses with $n$ interior rolls disappear in fold bifurcations: the branches for different values of $n$ disappear in the ordering given by $n$, with branches for smaller $n$ vanishing first. This indicates that we should be able to find worms with a small number of interior rolls only for large values of $\nu$. Figures 29 and 30 contain continuation results of worms for $\nu=3$ and $\nu=2$, respectively, that start from radial spots. While the worms in Figure 29 contain only a few rolls, the patterns shown in Figure 30 have a large number of interior rolls as predicted by Figure 28.

It is natural to ask how the branches of fully localised stripe patterns acquire additional fold bifurcations as we decrease $\nu$ or, in other words, how the branches shown in Figures 29 and 30 transform into each other upon decreasing $\nu$. It turns out that the mechanism is quite complicated and, interestingly, involves isolated branches such as the rightmost branch from Figure 24. To illustrate the creation of new folds, we present in Figure 31 continuation results for two nearby values of $\nu$. We see that the snaking branch and a second isolated branch collide and rearrange themselves, leading to an additional fold bifurcation, and therefore an increase of the number of rolls inside the fully localised structures, along the snaking branch. When $\nu$ is decreased further, the rightmost blue branch in the right panel of Figure 31 moves towards the left and 

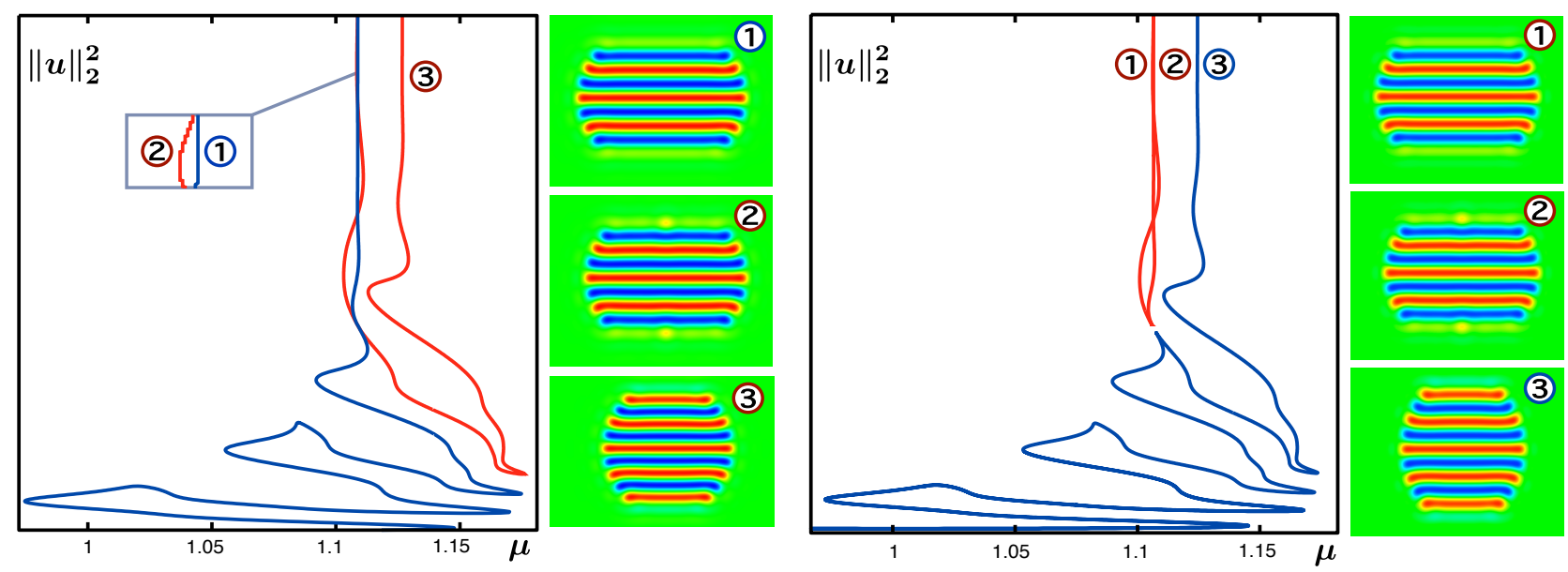

Figure 31: Two branches of fully localised stripe patterns are shown for $\nu=2.655$ (left) and $\nu=2.652$ (right). The blue snaking branch and the leftmost branch of the red isola in the left panel collide and rearrange themselves upon decreasing $\nu$, leading to the red isola and the blue snaking branch shown in the right panel. As a result, the emerging blue branch in the right panel snakes for longer. The numerical parameters are $L_{x}=L_{y}=90$ and $n_{x}=n_{y}=220$.
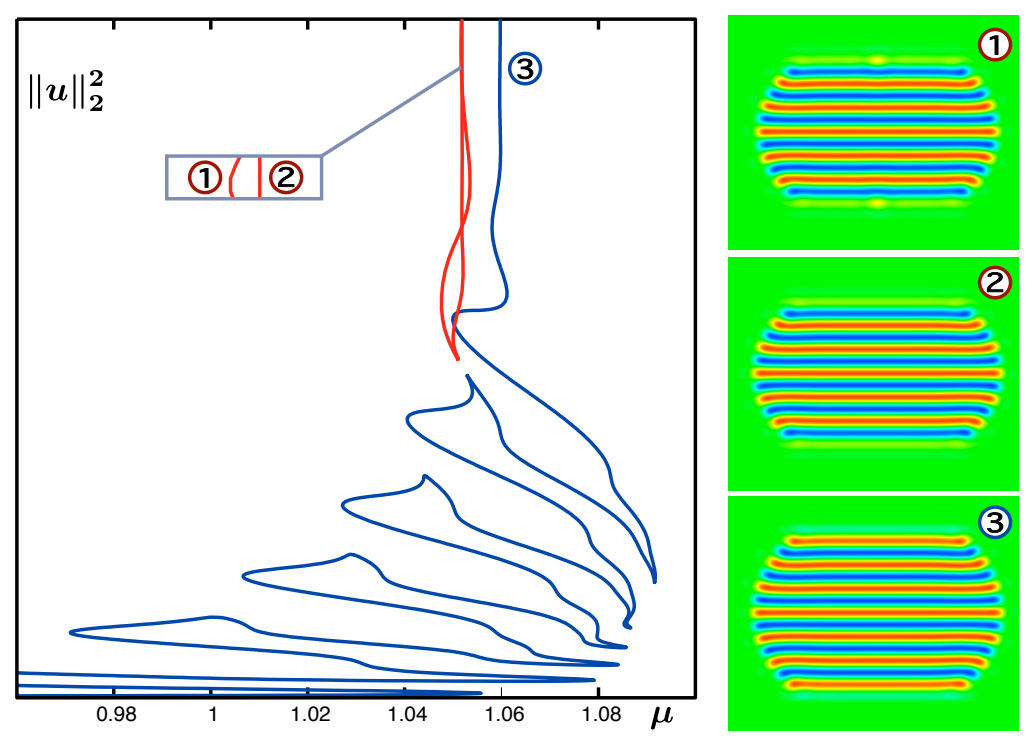

Figure 32: The two branches of fully localised stripe patterns from Figure 31 are shown for $\nu=2.556$. The bifurcation structure shown here indicates that the process of collision and subsequent rearrangement of branches that we observed in Figure 31 repeats itself upon decreasing $\nu$ further, thus leading to more extended snaking curves such as the one plotted here. The numerical parameters are $L_{x}=L_{y}=90$ and $n_{x}=n_{y}=220$.

eventually collides again with the left-most red branch, leading to a rearrangement similar to that shown in Figure 31. As indicated in Figure 32, this mechanism of adding additional folds along the branch seems to continue indefinitely upon decreasing $\nu$ further. We remark that the constant changes of the relative position of the different branches under variations of $\nu$ make it difficult to compute them using direct continuation in $\nu$. Instead, we continued fold bifurcation curves in the parameters $(\mu, \nu)$ and afterwards restarted continuation in $\mu$ from these folds for different fixed values of $\nu$.

The pattern shown in Figure 30 for $\nu=2$ looks quite different from the worm patches shown in the other figures for larger values of $\nu$ : the top and bottom caps of the patterns for $\nu=2$ are peaked instead of flat. Figure 33 and the accompanying movie illustrate this spatial behaviour further. In particular, the sign of the curvature near the peaked top and bottom ends of the worm patches seems to change along the snaking branch. This phenomenon is not explained by our spatial-dynamics interpretation, and we discuss it further in $\S 6$. 


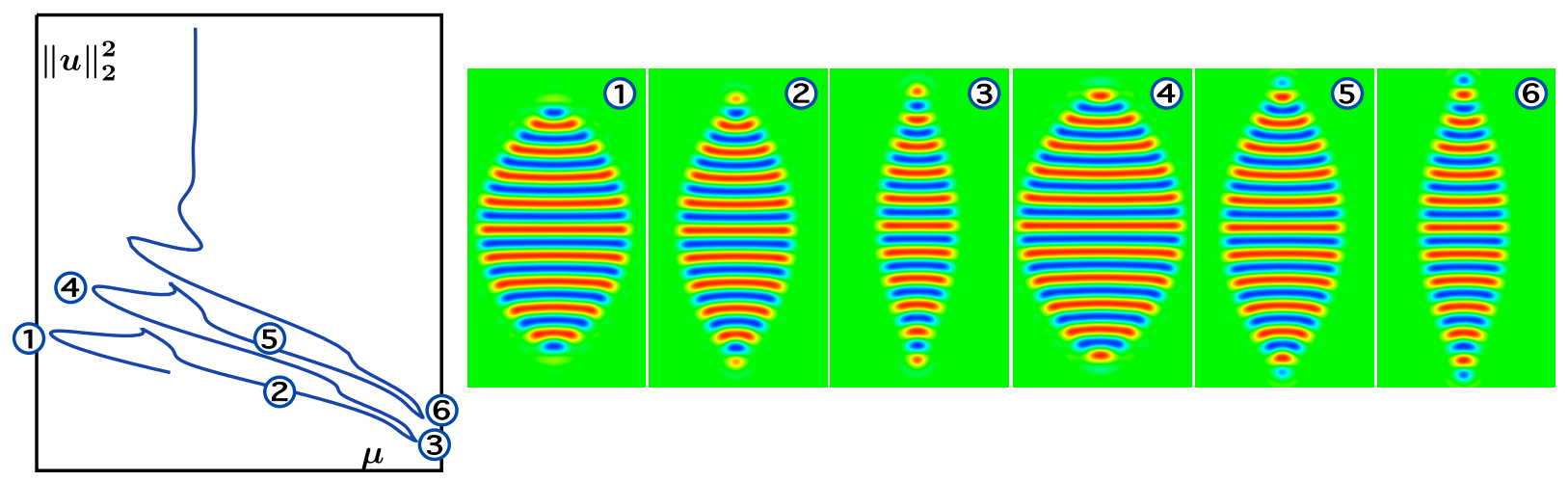

Figure 33: The continuation results for $\nu=2.46$ presented here illustrate how the curvature of the worm boundary near its peaked top and bottom end points changes along the solution branch: the sign of the curvature of the localised patterns in panels (3) and (6) near its peaked end points is different from those in the other panels. The family of worm profiles along the bifurcation curve can also be viewed in the accompanying movie. The computation was done with $L_{x}=88, L_{y}=157, n_{x}=257$, and $n_{y}=1025$.

\section{Discussion}

In this paper, we presented a comprehensive numerical study of localised stripe patterns for the planar cubicquintic Swift-Hohenberg equation. The main motivation was our desire to connect the profiles of planar patterns and the shape of their bifurcation branches to relatively simple ideas from dynamical systems theory. We hope that we convinced the reader that these ideas can explain snaking and non-snaking of solution branches and elucidate the mechanisms that lead to changes of the profiles along these branches. We have also seen, however, that there are many questions that cannot, at this stage, been addressed using spatial dynamics, and in the remainder of this section we outline some of the remaining issues together with other open problems.

Spatial dynamics is most predictive when there is one distinguished direction in which patterns grow. A particular example are patterns that are periodic in one variable and localised in the other spatial direction, for which our results in $\S 4$ give excellent agreement with abstract theoretical predictions, primarily because we can represent the underlying partial differential equation uniquely by a dynamical system in the unbounded variable. Fully localised patterns, on the other hand, may change in either of the two unbounded spatial variables as a parameter is varied, and there is no way of capturing these changes by a single spatial dynamical system. Indeed, the fully localised hexagon patches studied in [30] exhibit a very complex bifurcation structure, with equally complex profile changes along their solution branch. The fully localised stripe patches we considered in $\S 5$ behave somewhere in between these extremes as the rolls they are comprised of select two distinct directions along which changes are preferred. The two different growth mechanisms in the direction along and transverse to the roll patterns are reflected in the spatial-dynamics interpretation we put forward in Figure 25, which, in turn, explained some of the features of worms we encountered during their numerical continuation.

However, these ideas cannot explain the precise shape of fully localised stripe patterns or the changes in curvature of the spatial interface between background and interior roll structures that are visible along the branch shown in Figure 33. These indicate that simple notions based on effective surface "tension" are insufficient to determine the shape of the structure, and suggest that the shape is defined by the competition between surface energy and changes in the bulk energy due to curvature-induced wavelength variation. Work along these lines includes that of [19] who use modulation equations to capture weakly nonlinear interfaces between a background and a hexagonal pattern to describe the possible shapes of the fully localised hexagonal structures computed in [30] for the planar Swift-Hohenberg equation. Similar structures are present in nonvariational systems as well [23] although for such systems energy-based methods are of doubtful value. A similar approach may be useful for elucidating the shape of worms, at least in the context of the planar 
Swift-Hohenberg equation. A possible alternative approach using Wulff diagrams that are used to predict the shape of crystals through appropriate interfacial energies may also be useful.

In most of our computations, fully localised stripe patterns cease to snake once their $L^{2}$-norm becomes large enough, although the results shown in Figure 30 are inconclusive in this regard. We speculate that worm branches will always stop snaking and instead approach a vertical asymptote but have no proof to offer.

Finally, we mention the implications that our results may have for applications. Our computations for almost planar stripe patterns indicate that the asymmetric states created by the underlying bifurcation can be temporally stable. This is rather unexpected and raises, for instance, the possibility of finding stable asymmetric localised buckling modes of the cylindrical shells analysed in [22]. Time-independent worm-like states of the type computed here should also be present in non-variational systems such as convection with midplane reflection symmetry [4] or plane Couette flow [41], although their stability properties will differ.

Acknowledgments. Avitabile was supported by the EPSRC through the grant Applied Nonlinear Mathematics: Making it Real (EP/E032249/1). This work was also supported in part by a Royal Society-Wolfson Research Merit Award to Sandstede and by the NSF through grants DMS-0602204 (Burke), DMS-0908102 (Knobloch) and DMS-0907904 (Sandstede).

\section{References}

[1] H. Arbell and J. Fineberg. Temporally harmonic oscillons in Newtonian fluids. Phys. Rev. Lett. 85 (2000) 756-759.

[2] Y. Astrov and Y. Logvin. Formation of clusters of localized states in a gas discharge system via a self-completion scenario. Phys. Rev. Lett. 79 (1997) 2983-2986.

[3] D. Avitabile. Computation of planar patterns and their stability. Ph.D. thesis, University of Surrey, 2008.

[4] O. Batiste, E. Knobloch, A. Alonso and I. Mercader. Spatially localized binary-fluid convection. J. Fluid Mech. 560 (2006) 149-158.

[5] M. Beck, J. Knobloch, D. Lloyd, B. Sandstede and T. Wagenknecht. Snakes, ladders, and isolas of localised patterns. SIAM J. Math. Anal. 41 (2009) 936-972.

[6] U. Bisang and G. Ahlers. Bifurcation to worms in electroconvection. Phys. Rev. E 60 (1999) 3910-3919.

[7] U. Bortolozzo, M. G. Clerc and S. Residori. Solitary localized structures in a liquid crystal light-valve experiment. New J. Phys. 11 (2009) 093037.

[8] J. Burke and E. Knobloch. Localized states in the generalized Swift-Hohenberg equation. Phys. Rev. E 73 (2006) 056211.

[9] J. Burke and E. Knobloch. Homoclinic snaking: Structure and stability. Chaos 17 (2007) 037102.

[10] J. Burke and E. Knobloch. Snakes and ladders: localized states in the Swift-Hohenberg equation. Phys. Lett. A 360 (2007) 681-688.

[11] A. R. Champneys and B. Sandstede. Numerical computation of coherent structures. In: Numerical Continuation Methods for Dynamical Systems (B. Krauskopf, H. M. Osinga and J. Galan-Vioque, eds.). Springer, 2007, 331-358.

[12] S. J. Chapman and G. Kozyreff. Exponential asymptotics of localized patterns and snaking bifurcation diagrams. Physica D 238 (2009) 319-354. 
[13] P. Coullet, C. Riera and C. Tresser. Stable static localized structures in one dimension. Phys. Rev. Lett. 84 (2000) 3069-3072.

[14] J. D. Crawford and E. Knobloch. Symmetry and symmetry-breaking bifurcations in fluid mechanics. Ann. Rev. Fluid Mech. 23 (1991) 341-387.

[15] M. Cross and P. Hohenberg. Pattern formation outside of equilibrium. Rev. Mod. Phys. 65 (1993) $851-1112$.

[16] F. Daviaud, J. Hegseth and P. Bergé. Subcritical transition to turbulence in plane Couette flow. Phys. Rev. Lett. 69 (1992) 2511-2514.

[17] M. Dennin, G. Ahlers and D. S. Cannell. Chaotic localized states near the onset of electroconvection. Phys. Rev. Lett. 77 (1996) 2475-2478.

[18] E. J. Doedel and B. Oldeman. AUTO07P: continuation and bifurcation software for ordinary differential equations. Technical report, Concordia University, 2009.

[19] D. Escaff and O. Descalzi. Shape and size effects in localized hexagonal patterns. Internat. J. Bifur. Chaos Appl. Sci. Engrg. 19 (2009) 2727-2743.

[20] M. A. Heroux, R. A. Bartlett, V. E. Howle, R. J. Hoekstra, J. J. Hu, T. G. Kolda, R. B. Lehoucq, K. R. Long, R. P. Pawlowski, E. T. Phipps, A. G. Salinger, H. K. Thornquist, R. S. Tuminaro, J. M. Willenbring, A. Williams and K. S. Stanley. An overview of the Trilinos project. ACM Trans. Math. Softw. 31 (2005) 397-423.

[21] R. B. Hoyle. Pattern Formation. Cambridge University Press, Cambridge, 2006.

[22] G. W. Hunt, M. A. Peletier, A. R. Champneys, P. D. Woods, M. A. Wadee, C. J. Budd and G. J. Lord. Cellular buckling in long structures. Nonlin. Dynam. 21 (2000) 3-29.

[23] O. Jensen, V. O. Pannbacker, E. Mosekilde, G. Dewel and P. Borckmans. Localized structures and front propagation in the Lengyel-Epstein model. Phys. Rev. E 50 (1994) 736-749.

[24] A. Joets and R. Ribotta. Localized, time-dependent state in the convection of a nematic liquid crystal. Phys. Rev. Lett. 60 (1988) 2164-2167.

[25] J. Knobloch and T. Wagenknecht. Homoclinic snaking near a heteroclinic cycle in reversible systems. Physica D 206 (2005) 82-93.

[26] P. Kolodner. Coexisting traveling waves and steady rolls in binary-fluid convection. Phys. Rev. E 48 (1993) R665-668.

[27] G. Kozyreff and S. J. Chapman. Asymptotics of large bound states of localized structures. Phys. Rev. Lett. 97 (2006) 044502.

[28] K.-J. Lee, W. D. McCormick, J. E. Pearson and H. L. Swinney. Experimental observation of selfreplicating spots in a reaction-diffusion system. Nature 369 (1996) 215-218.

[29] O. Lioubashevski, Y. Hamiel, A. Agnon, Z. Reches and J. Fineberg. Oscillons and propagating solitary waves in a vertically vibrated colloidal suspension. Phys. Rev. Lett. 83 (1999) 756-759.

[30] D. J. B. Lloyd, B. Sandstede, D. Avitabile and A. R. Champneys. Localized hexagon patterns of the planar Swift-Hohenberg equation. SIAM J. Appl. Dynam. Syst. 7 (2008) 1049-1100.

[31] D. Peterhof, B. Sandstede and A. Scheel. Exponential dichotomies for solitary-wave solutions of semilinear elliptic equations on infinite cylinders. J. Diff. Eqns. 140 (1997) 266-308. 
[32] S. Pirkl, P. Ribière and P. Oswald. Forming process and stability of bubble domains in dielectrically positive cholesteric liquid crystals. Liquid Crystals 13 (1993) 413-415.

[33] L. M. Pismen. Patterns and Interfaces in Dissipative Dynamics. Springer-Verlag, Berlin, 2006.

[34] Y. Pomeau. Front motion, metastability, and subcritical bifurcations in hydrodynamics. Physica D 23 (1986) 3-11.

[35] R. Richter and I. V. Barashenkov. Two-dimensional solitons on the surface of magnetic fluids. Phys. Rev. Lett. 94 (2005) 184503.

[36] H. Sakaguchi and H. R. Brand. Stable localized solutions of arbitrary length for the quintic SwiftHohenberg equation. Physica D 97 (1996) 274-285.

[37] B. Sandstede and A. Scheel. Defects in oscillatory media: toward a classification. SIAM J. Appl. Dyn. Syst. 3 (2004) 1-68.

[38] B. Sandstede and A. Scheel. Relative Morse indices, Fredholm indices, and group velocities. Discr. Cont. Dyn. Syst. A 20 (2008) 139-158.

[39] B. Schäpers, M. Feldmann, T. Ackemann and W. Lange. Interaction of localized structures in an optical pattern-forming system. Phys. Rev. Lett. 85 (2000) 748-751.

[40] F. Schilder. EPCOnT: A continuation toolbox for Matlab. Personal communication (2007).

[41] T. M. Schneider, J. F. Gibson and J. Burke. Snakes and ladders: localized solutions of plane Couette flow. Phys. Rev. Lett. 104 (2010) 104501.

[42] N. Tillmark and P. H. Alfredsson. Experiments on transition in plane Couette flow. J. Fluid Mech. 235 (1992) 89-102.

[43] L. N. Trefethen. Spectral Methods in MatLaB. SIAM, Philadelphia, 2000.

[44] P. B. Umbanhowar, F. Melo and H. L. Swinney. Localised excitations in a vertically vibrated granular layer. Nature 382 (1996) 793-796.

[45] P. D. Woods and A. R. Champneys. Heteroclinic tangles and homoclinic snaking in the unfolding of a degenerate reversible Hamiltonian-Hopf bifurcation. Physica D 129 (1999) 147-170. 